\title{
On State-Dependent Delay Intuitionistic Fuzzy Partial Functional Differential Equations with Integral Boundary Conditions
}

\author{
Bouchra Ben Amma (D), Said Melliani D, and Lalla Saadia Chadli \\ Laboratory of Applied Mathematics and Scientific Computing, Faculty of Sciences and Technologies, \\ Sultan Moulay Slimane University, BP 523, 23000 Beni Mellal, Morocco
}

Correspondence should be addressed to Bouchra Ben Amma; bouchrabenamma@gmail.com

Received 10 May 2021; Accepted 14 June 2021; Published 5 July 2021

Academic Editor: Niansheng Tang

Copyright (c) 2021 Bouchra Ben Amma et al. This is an open access article distributed under the Creative Commons Attribution License, which permits unrestricted use, distribution, and reproduction in any medium, provided the original work is properly cited.

\begin{abstract}
This paper addresses the issue of the existence and uniqueness of intuitionistic fuzzy solutions for some classes of partial functional differential equations with state-dependent delay in a new weighted complete metric space. Theorems on the existence and uniqueness of intuitionistic fuzzy solutions for these problems with integral boundary conditions are established under some sufficient assumptions. Some numerical examples of applications of the main result of this work are presented.
\end{abstract}

\section{Introduction}

In 1965, Zadeh [1] proposed the concept of fuzzy sets with the purpose to model ambiguity, uncertainty, and vagueness in complicated systems. It can be considered as an extension of the usual (crisp) set theory. It has greater pliability to capture different aspects of incompleteness, imperfection, and uncertainty in data about many situations. The membership $\mu$ of an element of a fuzzy set is a single value between 0 and 1 . Therefore, in reality, it may not always be true that the degree of nonmembership $v$ of an element in a fuzzy set is equal to 1 minus the membership degree $(1-\mu)$ because there may be some uncertainty degree. Thus, since the fuzzy set has no means to characterize the neutral state, neither support nor oppose, Atanassov [2] included the nonmembership function and defined the degree of uncertainty as $1-\mu-\nu$. He introduced the topic of intuitionistic fuzzy sets (IFSs) as an extension of the standard fuzzy sets [3]. Several applications of IFS theory in diverse fields have been carried out; they are a very necessary and powerful tool in modeling imprecision; IFSs have been flourished in many different fields, an intuitionistic fuzzy approach to artificial intelligence $[4,5]$, medical diagnosis [6], drug selection [7], along with pattern recognition [8], microelectronic fault analysis [9], and decision-making problems $[10,11]$.
The concept of intuitionistic fuzzy differential equations has a rich history and is one of the actively developing topics of intuitionistic fuzzy set theory. The first paper on intuitionistic fuzzy differential equations was by Melliani and Chadli [12], and recently, the authors established some intuitionistic fuzzy solutions for such equations. They proved the existence and uniqueness of intuitionistic fuzzy solutions under some assumptions for these intuitionistic fuzzy differential equations using different procedures, see $[13,14]$. The topic of intuitionistic fuzzy functional differential equations with delay is very rare: in [15], they first elaborated conditions that can be satisfied by intuitionistic fuzzy functional differential equations with delay and that guarantee the existence and uniqueness of local and global intuitionistic fuzzy solutions for such equations. Numerical algorithms for treatment of IFDEs are still evolving, see [16-20] for applications.

The notion of intuitionistic fuzzy partial differential equations was first studied in 2000 [21], and intuitionistic fuzzy partial differential equations with integral boundary conditions were first introduced in 2019 [22], where they proved existence and uniqueness results and defined a procedure to calculate the intuitionistic fuzzy solutions of such problems. However, according to our knowledge, research on the properties of solutions of intuitionistic fuzzy 
partial functional differential equations is very limited. The study of intuitionistic fuzzy partial differential equations with state-dependent delay has been initiated recently in 2019 [23], in which they considered the existence and uniqueness of intuitionistic fuzzy solutions for these types of equations with local, nonlocal, and integral boundary conditions, based on some complicated assumptions on the domain. But those hypotheses can be reduced to be milder. In the present paper, our models can be considered as an extension and complementation of the previous results into new weighted metric spaces of intuitionistic fuzzy valued functions. We study state-dependent delay intuitionistic fuzzy partial functional differential equations with integral boundary conditions of the following forms:

$$
\begin{aligned}
& \begin{cases}\frac{\partial^{2}\left\langle\phi_{1}, \phi_{2}\right\rangle(\lambda, \theta)}{\partial \lambda \partial \theta}=G_{1}\left(\lambda, \theta,\left\langle\phi_{1}, \phi_{2}\right\rangle(\lambda, \theta),\right. & (\lambda, \theta) \in I_{c} \times I_{d}, \\
\left\langle\phi_{1}, \phi_{2}\right\rangle(\lambda, 0)+\int_{0}^{\theta} L_{1}(\lambda)\left\langle\phi_{1}, \phi_{2}\right\rangle(\lambda, \sigma) \mathrm{d} \sigma=f_{1}(\lambda, \theta), & (\lambda, \theta) \in I_{c} \times I_{d}, \\
\left\langle\phi_{1}, \phi_{2}\right\rangle(0, \theta)+\int_{0}^{\lambda} L_{2}(\theta)\left\langle\phi_{1}, \phi_{2}\right\rangle(\zeta, \theta) \mathrm{d} \zeta=f_{2}(\lambda, \theta), & (\lambda, \theta) \in I_{c} \times I_{d},\end{cases} \\
& \begin{cases}\frac{\partial^{2}\left\langle\phi_{1}, \phi_{2}\right\rangle(\lambda, \theta)}{\partial \lambda \partial \theta}=\frac{\partial\left(q(\lambda, \theta)\left\langle\phi_{1}, \phi_{2}\right\rangle(\lambda, \theta)\right)}{\partial \theta}+G_{2}\left(\lambda, \theta,\left\langle\phi_{1}, \phi_{2}\right\rangle(\lambda, \theta),\right. & (\lambda, \theta) \in I_{c} \times I_{d}, \\
\left\langle\phi_{1}, \phi_{2}\right\rangle(\lambda, 0)+\int_{0}^{\theta} L_{1}(\lambda)\left\langle\phi_{1}, \phi_{2}\right\rangle(\lambda, \sigma) \mathrm{d} \sigma=f_{1}(\lambda, \theta), & (\lambda, \theta) \in I_{c} \times I_{d}, \\
\left\langle\phi_{1}, \phi_{2}\right\rangle(0, \theta)+\int_{0}^{\lambda} L_{2}(\theta)\left\langle\phi_{1}, \phi_{2}\right\rangle(\zeta, \theta) \mathrm{d} \zeta=f_{2}(\lambda, \theta), & (\lambda, \theta) \in I_{c} \times I_{d},\end{cases}
\end{aligned}
$$

where $G_{1}, G_{2}: I_{c} \times I_{d} \times \mathrm{IF}_{n} \longrightarrow \mathrm{IF}_{n}$ is continuous and $q \in C\left(I_{c} \times I_{d}, \mathbb{R}\right), L_{1} \in C\left(I_{c}, \mathbb{R}\right), L_{2} \in C\left(I_{d}, \mathbb{R}\right), f_{1} \in C\left(I_{c} \times\right.$ $\left.I_{d}, \mathrm{IF}_{n}\right)$, and $f_{2} \in C\left(I_{c} \times I_{d}, \mathrm{IF}_{n}\right)$ are given functions.

Motivated by this consideration, first, we shall discuss the existence and uniqueness of the intuitionistic fuzzy solutions to those problems by using some appropriate Banach fixed point theorems under suitable conditions. The main contributions of this work are as follows: introducing the integral boundary value problem for intuitionistic fuzzy partial differential equations with the state-dependent delays and defining their solution; further developing theoretical results on the existence and uniqueness of the intuitionistic fuzzy solution; defining the intuitionistic fuzzy solution to these problems with integral boundary conditions through a corresponding parametric problem.

Delay differential equations have been used in many fields for a long time. Researchers have considered statedependent delay differential equations (SDDEs) for the last 50 years. However, more precisely integral initial boundary value problems with state-dependent delays constitute a very interesting and important class of applied problems; they have been used in modeling scientific phenomena in various applications in different fields such as biology, neural networks, control theory, physics, and medicine, see [24-33], for details. Linking the two aspects introduced, intuitionistic fuzzy mathematics and partial functional differential equations with state-dependent delays, we obtain intuitionistic fuzzy partial functional differential equations (IFPFDEs) with state-dependent delays, which will attract great interest both in mathematics and in applications. No result has been reported on the existing models of state-dependent delay IFPFDEs with integral boundary initial conditions, and among the purposes of this work is to fill this gap. This paper initiates the study of such equations: solving state-dependent delay IFPFDEs with integral boundary initial conditions, providing some definitions for intuitionistic fuzzy solutions of such problems, progressing the main results on the existence and uniqueness of intuitionistic fuzzy solutions under suitable hypothesizes in new weighted metric spaces, without any restraints in the data, displaying rigorous wellposedness and regularity analysis of the state-dependent delay intuitionistic fuzzy PFDEs, and establishing numerical examples that can be considered as a contribution to the subject of our present work and will be introduced elsewhere.

The rest of the paper is organised as follows: in Section 2, we will recall some basic definitions and preliminary facts which will be used throughout the following sections. In Section 3, we provide the existence and uniqueness of intuitionistic fuzzy solutions for partial functional differential equations with integral boundary conditions under suitable conditions in a new weighted metric space. In Section 4, we illustrate our abstract results by numerical applications. Finally, Section 5 gives the main conclusions and possible future works in this area. 


\section{Preliminaries}

We define $\mathrm{IF}_{n}$ as follows:

$$
\begin{aligned}
\operatorname{IF}_{n}= & \operatorname{IF}\left(\mathbb{R}^{n}\right) \\
= & \left\{\left\langle\phi_{1}, \phi_{2}\right\rangle: \mathbb{R}^{n} \longrightarrow[0,1] \times[0,1],\right. \\
& \left.\quad \mid \forall \lambda \in \mathbb{R}^{n}, 0 \leq \phi_{1}(\lambda)+\phi_{2}(\lambda) \leq 1\right\} .
\end{aligned}
$$

We called $\left\langle\phi_{1}, \phi_{2}\right\rangle \in \mathrm{IF}_{n}$ as an intuitionistic fuzzy number if it verifies the following assumptions:

(a) $\left\langle\phi_{1}, \phi_{2}\right\rangle$ is normal, i.e., there exists $\lambda_{0}$ and $\lambda_{1} \in \mathbb{R}^{n}$ such that $\phi_{1}\left(\lambda_{0}\right)=1$ and $\phi_{1}=2\left(\lambda_{1}\right)=1$

(b) $\phi_{1}$ is fuzzy convex, and $\phi_{2}$ is fuzzy concave

(c) $\phi_{1}$ is upper semicontinuous, and $\phi_{2}$ is lower semicontinuous (d) $\operatorname{supp}\left\langle\phi_{1}, \phi_{2}\right\rangle=c l\left\{\lambda \in \mathbb{R}^{n}: \mid \phi_{2}(\lambda)<1\right\}$ is bounded

We define the upper and lower $\alpha$-cuts of $\left\langle\phi_{1}, \phi_{2}\right\rangle \in \mathrm{IF}_{n}$ with $\alpha \in[0,1]$ by the following forms:

$$
\begin{array}{ll}
{\left[\left\langle\phi_{1}, \phi_{2}\right\rangle\right]^{\alpha}=\left\{\lambda \in \mathbb{R}^{n}:\right.} & \left.\phi_{2}(\lambda) \leq 1-\alpha\right\}, \\
{\left[\left\langle\phi_{1}, \phi_{2}\right\rangle\right]_{\alpha}=\left\{\lambda \in \mathbb{R}^{n}:\right.} & \left.\phi_{1}(\lambda) \geq \alpha\right\} .
\end{array}
$$

We define $0_{(1,0)} \in \mathrm{IF}_{n}$ as

$$
0_{(1,0)}(s)= \begin{cases}(1,0), & s=0, \\ (0,1), & s \neq 0 .\end{cases}
$$

Let $\left\langle\phi_{1}, \phi_{2}\right\rangle$ and $\left\langle\phi_{1}^{\prime}, \phi_{2}^{\prime}\right\rangle \in \mathrm{IF}_{n}$ and $\zeta \in \mathbb{R}$; then,

$$
\begin{aligned}
\left(\left\langle\phi_{1}, \phi_{2}\right\rangle+\left\langle\phi_{1}^{\prime}, \phi_{2}^{\prime}\right\rangle\right)(r) & =\left(\sup _{r=m+n} \min \left(\phi_{1}(m), \phi_{1}^{\prime}(n)\right), \inf _{r=m+n} \max \left(\phi_{2}(m), \phi_{2}^{\prime}(n)\right)\right), \\
\zeta\left\langle\phi_{1}, \phi_{2}\right\rangle & = \begin{cases}\langle\zeta v, \zeta w\rangle, & \text { if } \zeta \neq 0, \\
0_{(1,0)}, & \text { if } \zeta=0 .\end{cases}
\end{aligned}
$$

For $\left\langle\phi_{1}, \phi_{2}\right\rangle$ and $\left\langle\phi_{1}^{\prime}, \phi_{2}^{\prime}\right\rangle \in \mathrm{IF}_{n}$ and $\zeta \in \mathbb{R}$, therefore

$$
\begin{aligned}
& {\left[\left\langle\phi_{1}, \phi_{2}\right\rangle+\left\langle\phi_{1}^{\prime}, \phi_{2}^{\prime}\right\rangle\right]^{\alpha} }=\left[\left\langle\phi_{1}, \phi_{2}\right\rangle\right]^{\alpha}+\left[\left\langle\phi_{1}^{\prime}, \phi_{2}^{\prime}\right\rangle\right]^{\alpha}, \\
& {\left[\zeta\left\langle\phi_{1}, \phi_{2}\right\rangle\right]^{\alpha} }=\zeta\left[\left\langle\phi_{1}, \phi_{2}\right\rangle\right]^{\alpha}, \\
& {\left[\left\langle\phi_{1}, \phi_{2}\right\rangle+\left\langle\phi_{1}^{\prime}, \phi_{2}^{\prime}\right\rangle\right]_{\alpha}=\left[\left\langle\phi_{1}, \phi_{2}\right\rangle\right]_{\alpha}+\left[\left\langle\phi_{1}^{\prime}, \phi_{2}^{\prime}\right\rangle\right]_{\alpha}, } \\
& {\left[\zeta\left\langle\phi_{1}, \phi_{2}\right\rangle\right]_{\alpha}=\zeta\left[\left\langle\phi_{1}, \phi_{2}\right\rangle\right]_{\alpha} . }
\end{aligned}
$$

Definition 1. Let $\alpha \in[0,1]$ and $\left\langle\phi_{1}^{\prime}, \phi_{2}^{\prime}\right\rangle \in \mathrm{IF}_{n}$; the following sets are defined by

$$
\begin{aligned}
& {\left[\left\langle\phi_{1}, \phi_{2}\right\rangle\right]_{l}^{+}(\alpha)=\inf \left\{\lambda \in \mathbb{R}^{n} \mid \phi_{1}(\lambda) \geq \alpha\right\},} \\
& {\left[\left\langle\phi_{1}, \phi_{2}\right\rangle\right]_{r}^{+}(\alpha)=\sup \left\{\lambda \in \mathbb{R}^{n} \mid \phi_{1}(\lambda) \geq \alpha\right\},} \\
& {\left[\left\langle\phi_{1}, \phi_{2}\right\rangle\right]_{l}^{-}(\alpha)=\inf \left\{\lambda \in \mathbb{R}^{n} \mid \phi_{2}(\lambda) \leq 1-\alpha\right\},} \\
& {\left[\left\langle\phi_{1}, \phi_{2}\right\rangle\right]_{r}^{-}(\alpha)=\sup \left\{\lambda \in \mathbb{R}^{n} \mid \phi_{2}(\lambda) \leq 1-\alpha\right\} .}
\end{aligned}
$$

\section{Remark 1}

$$
\begin{aligned}
& {\left[\left\langle\phi_{1}, \phi_{2}\right\rangle\right]_{\alpha}=\left[\left[\left\langle\phi_{1}, \phi_{2}\right\rangle\right]_{l}^{+}(\alpha),\left[\left\langle\phi_{1}, \phi_{2}\right\rangle\right]_{r}^{+}(\alpha)\right],} \\
& {\left[\left\langle\phi_{1}, \phi_{2}\right\rangle\right]^{\alpha}=\left[\left[\left\langle\phi_{1}, \phi_{2}\right\rangle\right]_{l}^{-}(\alpha),\left[\left\langle\phi_{1}, \phi_{2}\right\rangle\right]_{r}^{-}(\alpha)\right] .}
\end{aligned}
$$

Proposition 1 (see [34]). For all $\alpha_{1}$ and $\alpha_{2} \in[0,1]$ and $\left\langle\phi_{1}, \phi_{2}\right\rangle \in I F_{n}$,

(a) $\left[\left\langle\phi_{1}, \phi_{2}\right\rangle\right]_{\alpha_{1}} \subset\left[\left\langle\phi_{1}, \phi_{2}\right\rangle\right]^{\alpha_{1}}$ (b) $\left[\left\langle\phi_{1}, \phi_{2}\right\rangle\right]_{\alpha_{1}}$ and $\left[\left\langle\phi_{1}, \phi_{2}\right\rangle\right]^{\alpha_{1}}$ are nonempty compact convex sets in $\mathbb{R}^{n}$

(c) if $\alpha_{1} \leq \alpha_{2}$, therefore $\left[\left\langle\phi_{1}, \phi_{2}\right\rangle\right]_{\alpha_{2}} \subset\left[\left\langle\phi_{1}, \phi_{2}\right\rangle\right]_{\alpha_{1}}$ and $\left[\left\langle\phi_{1}, \phi_{2}\right\rangle\right]^{\alpha_{2}} \subset\left[\left\langle\phi_{1}, \phi_{2}\right\rangle\right]^{\alpha_{1}}$

(d) If $\alpha_{n} \nearrow \alpha_{1}$, therefore $\left[\left\langle\phi_{1}, \phi_{2}\right\rangle\right]_{\alpha_{1}}=\cap_{n}\left[\left\langle\phi_{1}, \phi_{2}\right\rangle\right]_{\alpha_{n}}$ and $\left[\left\langle\phi_{1}, \phi_{2}\right\rangle\right]^{\alpha_{1}}=\cap_{n}\left[\left\langle\phi_{1}, \phi_{2}\right\rangle\right]^{\alpha_{n}^{1}}$

Let $\alpha \in[0,1]$; we denote $N$ (any set) as follows:

$$
\begin{aligned}
& N_{\alpha}=\left\{\lambda \in \mathbb{R}^{n}: \phi_{1}(\lambda) \geq \alpha\right\}, \\
& N^{\alpha}=\left\{\lambda \in \mathbb{R}^{n}: \phi_{2}(\lambda) \leq 1-\alpha\right\} .
\end{aligned}
$$

Lemma 1 (see [34]). Let $\left\{N_{\alpha}, \alpha \in[0,1]\right\}$ and $\left\{N^{\alpha}, \alpha \in[0,1]\right\}$ be two families of subsets of $\mathbb{R}^{n}$; verify the assumptions of Proposition 1 if $\phi_{1}$ and $\phi_{2}$ are defined as follows:

$$
\begin{aligned}
& \phi_{1}(\lambda)= \begin{cases}0, & \text { if } \lambda \notin N_{0}, \\
\sup \left\{\alpha \in[0,1]: \lambda \in N_{\alpha}\right\}, & \text { if } \lambda \in N_{0},\end{cases} \\
& \phi_{2}(\lambda)= \begin{cases}1, & \text { if } \lambda \notin N^{0}, \\
1-\sup \left\{\alpha \in[0,1]: \lambda \in N^{\alpha}\right\}, & \text { if } \lambda \in N^{0} .\end{cases}
\end{aligned}
$$

Then, $\left\langle\phi_{1}, \phi_{2}\right\rangle \in \mathrm{IF}_{n}$.

We define the following metric by 


$$
\begin{aligned}
d_{\infty}^{n}\left(\left\langle\phi_{1}, \phi_{2}\right\rangle,\left\langle\phi_{1}^{\prime}, \phi_{2}^{\prime}\right\rangle\right)= & \frac{1}{4} \sup _{0<\alpha \leq 1}\left\|\left[\left\langle\phi_{1}, \phi_{2}\right\rangle\right]_{r}^{+}(\alpha)-\left[\left\langle\phi_{1}^{\prime}, \phi_{2}^{\prime}\right\rangle\right]_{r}^{+}(\alpha)\right\| \\
& +\frac{1}{4} \sup _{0<\alpha \leq 1}\left\|\left[\left\langle\phi_{1}, \phi_{2}\right\rangle\right]_{l}^{+}(\alpha)-\left[\left\langle\phi_{1}^{\prime}, \phi_{2}^{\prime}\right\rangle\right]_{l}^{+}(\alpha)\right\| \\
& +\frac{1}{4} \sup _{0<\alpha \leq 1}\left\|\left[\left\langle\phi_{1}, \phi_{2}\right\rangle\right]_{r}^{-}(\alpha)-\left[\left\langle\phi_{1}^{\prime}, \phi_{2}^{\prime}\right\rangle\right]_{r}^{-}(\alpha)\right\| \\
& +\frac{1}{4} \sup _{0<\alpha \leq 1}\left\|\left[\left\langle\phi_{1}, \phi_{2}\right\rangle\right]_{l}^{-}(\alpha)-\left[\left\langle\phi_{1}^{\prime}, \phi_{2}^{\prime}\right\rangle\right]_{l}^{-}(\alpha)\right\| .
\end{aligned}
$$

where $\|$.$\| is the usual Euclidean norm in \mathbb{R}^{n}$.

Theorem 1 (see [34]). $d_{\infty}^{n}$ is a metric on $I F_{n}$.

Theorem 2 (see [34]). (IF,$\left.d_{\infty}^{n}\right)$ is a complete metric space.

We denote

(i) $I_{c}=[0, c], \quad I_{d}=[0, d], \quad I_{0}=[-r, 0] \times[-r, 0]$, $I_{r}=[-r, c] \times[-r, d]$, and $\widehat{I}_{r}=I_{r} \backslash(0, c] \times(0, d]$

(ii) $C\left(I_{i}, \mathrm{IF}_{n}\right)$ : the space of all continuous mappings defined over $I_{i}$ into $\operatorname{IF}_{n}(i=c, d, 0$, and $r)$

For $\beta>0$ arbitrary, it can be shown that $\left(C\left(I_{r}, I_{n}\right), D_{\beta}\right)$ is a complete metric space, where the supremum weighted metric $D_{\beta}$ on $C\left(I_{r}, \mathrm{IF}_{n}\right)$ is defined by

$$
\begin{aligned}
& D_{\beta}\left(\left\langle\phi_{1}, \phi_{2}\right\rangle,\left\langle\phi_{1}^{\prime}, \phi_{2}^{\prime}\right\rangle\right) \\
& =\sup _{\left(r_{1}, r_{2}\right) \in I_{r}}\left\{d_{\infty}^{n}\left(\left\langle\phi_{1}, \phi_{2}\right\rangle\left(r_{1}, r_{2}\right),\left\langle\phi_{1}^{\prime}, \phi_{2}^{\prime}\right\rangle\left(r_{1}, r_{2}\right)\right) e^{-\beta\left(r_{1}+r_{2}\right)}\right\} .
\end{aligned}
$$

Definition 2. A mapping $G_{1}: I_{r} \longrightarrow \mathrm{IF}_{n}$ is continuous at point $\left(\lambda_{0}, \theta_{0}\right) \in I_{r}$ provided for any arbitrary $\varepsilon>0$; there exists an $\sigma(\varepsilon)$ such that

$$
d_{\infty}^{n}\left(G_{1}(\lambda, \theta), G_{1}\left(\lambda_{0}, \theta_{0}\right)\right)<\varepsilon,
$$

whenever $\max \left\{\left|\lambda-\lambda_{0}\right|,\left|\theta-\theta_{0}\right|\right\}<\sigma(\varepsilon) \forall(\lambda, \theta) \in I_{r}$.

Definition 3. A mapping $G_{1}: I_{r} \times \mathrm{IF}_{n} \longrightarrow \mathrm{IF}_{n}$ is called continuous at point $\left(\lambda_{0}, \theta_{0},\left\langle\phi_{1}, \phi_{2}\right\rangle_{0}\right) \in I_{r} \times \operatorname{IF}_{n}$ provided for any arbitrary $\varepsilon>0$; there exists an $\sigma(\varepsilon)$ such that

$$
d_{\infty}^{n}\left(G_{1}\left(\lambda, \theta,\left\langle\phi_{1}, \phi_{2}\right\rangle\right), G_{1}\left(\lambda_{0}, \theta_{0},\left\langle\phi_{1}, \phi_{2}\right\rangle_{0}\right)\right)<\varepsilon,
$$

whenever $\max \left\{\left|\lambda-\lambda_{0}\right|,\left|\theta-\theta_{0}\right|\right\}<\sigma(\varepsilon)$ and $d_{\infty}^{n}\left(\left\langle\phi_{1}, \phi_{2}\right\rangle\right.$, $\left.\left\langle\phi_{1}, \phi_{2}\right\rangle_{0}\right)<\sigma(\varepsilon) \forall(\lambda, \theta) \in I_{r},\left\langle\phi_{1}, \phi_{2}\right\rangle \in \mathrm{IF}_{n}$.

Definition 4. A mapping $G_{1}: I_{c} \times I_{d} \longrightarrow \mathrm{IF}_{n}$ is called strongly measurable if for $\forall \alpha \in[0,1]$, the set-valued mappings $g_{\alpha}: I_{c} \times I_{d} \longrightarrow P_{k}\left(\mathbb{R}^{n}\right)$ defined by $g_{\alpha}(\lambda, \theta)=$ $\left[G_{1}(\lambda, \theta)\right]_{\alpha}$ and $g^{\alpha}: I_{c} \times I_{d} \longrightarrow P_{k}\left(\mathbb{R}^{n}\right)$ defined by $g^{\alpha}(\lambda, \theta)=\left[G_{1}(\lambda, \theta)\right]^{\alpha}$ are (Lebesgue) measurable.

Definition 5. $G_{1}: I_{c} \times I_{d} \longrightarrow \mathrm{IF}_{n}$ is integrably bounded if there exists an integrable function $h: I_{c} \times I_{d} \longrightarrow \mathbb{R}^{n}$ such that $\|(\zeta, \sigma)\| \leq h(\lambda, \theta)$ holds for any $(\zeta, \sigma) \in \operatorname{supp}$ $\left(G_{1}(\lambda, \theta)\right),(\lambda, \theta) \in I_{c} \times I_{d}$.

Definition 6. Suppose $G_{1}: I_{c} \times I_{d} \longrightarrow \mathrm{IF}_{n}$ is integrably bounded and strongly measurable for each $\alpha \in(0,1]$; then,

$$
\begin{aligned}
{\left[\int_{0}^{c} \int_{0}^{d} G_{1}(\lambda, \theta) \mathrm{d} \theta \mathrm{d} \lambda\right]_{\alpha} } & =\int_{0}^{c} \int_{0}^{d}\left[G_{1}(\lambda, \theta)\right]_{\alpha} \mathrm{d} \theta \mathrm{d} \lambda \\
& =\left\{\int_{0}^{c} \int_{0}^{d} G(\lambda, \theta) \mathrm{d} \theta \mathrm{d} \lambda \mid G: I_{c} \times I_{d} \longrightarrow \mathbb{R}^{n} \text { is a measurable selection for } G_{1 \alpha}\right\} \\
{\left[\int_{0}^{c} \int_{0}^{d} G_{1}(\lambda, \theta) \mathrm{d} \theta \mathrm{d} \lambda\right]^{\alpha} } & =\int_{0}^{c} \int_{0}^{d}\left[G_{1}(\lambda, \theta)\right]^{\alpha} \mathrm{d} \theta \mathrm{d} \lambda \\
& =\left\{\int_{0}^{c} \int_{0}^{d} G(\lambda, \theta) \mathrm{d} \theta \mathrm{d} \lambda \mid G: I_{c} \times I_{d} \longrightarrow \mathbb{R}^{n} \text { is a measurable selection for } G_{1}^{\alpha}\right\}
\end{aligned}
$$

Let $G_{1}, G_{2}: I_{c} \times I_{d} \longrightarrow \mathrm{IF}_{n}$ be integrable and $k \in \mathbb{R}$. The integral has the elementary properties as follows: (i) $\int_{0}^{c} \int_{0}^{d}\left[G_{1}(\lambda, \theta)+G_{2}(\lambda, \theta)\right] \mathrm{d} \theta \mathrm{d} \lambda=\int_{0}^{c} \int_{0}^{d} G_{1}(\lambda, \theta) \mathrm{d} \theta$ $\mathrm{d} \lambda+\int_{0}^{c} \int_{0}^{d} G_{2}(\lambda, \theta) \mathrm{d} \theta \mathrm{d} \lambda$ 
(ii) $\int_{0}^{c} \int_{0}^{d} k G_{1}(\lambda, \theta) \mathrm{d} \theta \mathrm{d} \lambda=k \int_{0}^{c} \int_{0}^{d} G_{1} G_{1}(\lambda, \theta) \mathrm{d} \theta \mathrm{d} \lambda$

(iii) $d_{\infty}^{n}\left(\int_{0}^{c} \int_{0}^{d} G_{1}(\lambda, \theta) \mathrm{d} \theta \mathrm{d} \lambda, \int_{0}^{c} \int_{0}^{d} G_{2}(\lambda, \theta) \mathrm{d} \theta \mathrm{d} \lambda\right) \leq$ $\int_{0}^{c} \int_{0}^{d} d_{\infty}^{n}\left(G_{1}(\lambda, \theta), G_{2}(\lambda, \theta)\right) \mathrm{d} \theta \mathrm{d} \lambda$

Definition 7. Let $\left\langle\phi_{1}, \phi_{2}\right\rangle$ and $\left\langle\phi_{1}^{\prime}, \phi_{2}^{\prime}\right\rangle \in \mathrm{IF}_{n}$; we call the Hukuhara difference as an intuitionistic fuzzy number $\left\langle\varphi_{1}, \varphi_{2}\right\rangle \in \mathrm{IF}_{n}$; if it exists, such that

$$
\left\langle\phi_{1}, \phi_{2}\right\rangle-\left\langle\phi_{1}^{\prime}, \phi_{2}^{\prime}\right\rangle=\left\langle\phi_{1}, \phi_{2}\right\rangle \Longleftrightarrow\left\langle\phi_{1}, \phi_{2}\right\rangle=\left\langle\phi_{1}^{\prime}, \phi_{2}^{\prime}\right\rangle+\left\langle\phi_{1}, \phi_{2}\right\rangle \text {. }
$$

Definition 8. Let $G_{1}:[c, d] \longrightarrow \mathrm{IF}_{n}$ be differentiable, and suppose that the derivative $g^{\prime}$ is integrable over $[c, d]$; if the Hukuhara difference $G_{1}(d)-G_{1}(c)$ exists in $\mathrm{IF}_{n}$, then we have $\int_{c}^{d} G_{1}^{\prime}(t) \mathrm{d} t=G_{1}(d)-G_{1}(c)$.

Definition 9. Let $G_{1}: I_{c} \times I_{d} \longrightarrow \mathrm{IF}_{n}$. The intuitionistic fuzzy partial derivative of $g$ with respect to $x$ at the point $\left(\lambda_{0}, \theta_{0}\right) \in I_{c} \times I_{d}$ is the intuitionistic fuzzy quantity $\left(\partial G_{1}\left(\lambda_{0}, \theta_{0}\right) / \partial \lambda\right) \in \mathrm{IF}_{n}$ such that $\forall \triangle t>0$ sufficiently small, the H-differences $G_{1}\left(\lambda_{0}+\Delta t, \theta_{0}\right)-G_{1}\left(\lambda_{0}, \theta_{0}\right)$ and $G_{1}\left(\lambda_{0}, \theta_{0}\right)-G_{1}\left(\lambda_{0}-\Delta t, \theta_{0}\right)$ exist in $\mathrm{IF}_{n}$ and the limits

$$
\begin{aligned}
& \frac{\partial G_{1}\left(\lambda_{0}, \theta_{0}\right)}{\partial \lambda}=\lim _{\Delta t \longrightarrow 0^{+}} \frac{G_{1}\left(\lambda_{0}+\Delta t, \theta_{0}\right)-G_{1}\left(\lambda_{0}, \theta_{0}\right)}{\Delta t}, \\
& \frac{\partial G_{1}\left(\lambda_{0}, \theta_{0}\right)}{\partial \lambda}=\lim _{\Delta t \longrightarrow 0^{+}} \frac{G_{1}\left(\lambda_{0}, \theta_{0}\right)-G_{1}\left(\lambda_{0}-\Delta t, \theta_{0}\right)}{\Delta t} .
\end{aligned}
$$

The intuitionistic fuzzy partial derivative of $g$ with respect to $\theta$ at the point $\left(\lambda_{0}, \theta_{0}\right) \in I_{c} \times I_{d}$ and higher order of intuitionistic fuzzy partial derivative of $g$ are defined similarly.

\section{The Main Results}

We prove the existence-uniqueness of the intuitionistic fuzzy solution for the following intuitionistic fuzzy functional partial differential equation with integral boundary conditions:

$$
\begin{cases}\frac{\partial^{2}\left\langle\phi_{1}, \phi_{2}\right\rangle(\lambda, \theta)}{\partial \lambda \partial \theta}=G_{1}\left(\lambda, \theta,\left\langle\phi_{1}, \phi_{2}\right\rangle(\lambda, \theta)\right), & (\lambda, \theta) \in I_{c} \times I_{d}:=[0, c] \times[0, d], \\ \left\langle\phi_{1}, \phi_{2}\right\rangle(\lambda, \theta)=\eta(\lambda, \theta), & (\lambda, \theta) \in \widehat{I}_{r}, \\ \left\langle\phi_{1}, \phi_{2}\right\rangle(\lambda, 0)+\int_{0}^{\theta} L_{1}(\lambda)\left\langle\phi_{1}, \phi_{2}\right\rangle(\lambda, \sigma) \mathrm{d} \sigma=f_{1}(\lambda, \theta), & (\lambda, \theta) \in I_{c} \times I_{d}, \\ \left\langle\phi_{1}, \phi_{2}\right\rangle(0, \theta)+\int_{0}^{\lambda} L_{2}(\theta)\left\langle\phi_{1}, \phi_{2}\right\rangle(\zeta, \theta) \mathrm{d} \zeta=f_{2}(\lambda, \theta), & (\lambda, \theta) \in I_{c} \times I_{d} .\end{cases}
$$

Assume that $G_{1}: I_{r} \times C\left(I_{0}, \mathrm{IF}_{n}\right) \longrightarrow \mathrm{IF}_{n} \quad$ and $\eta \in C\left(I_{0}, \mathrm{IF}_{n}\right)$ is continuous; $L_{1} \in C\left(I_{c}, \mathbb{R}\right), L_{2} \in C\left(I_{d}, \mathbb{R}\right)$, $f_{1} \in C\left(I_{c} \times I_{d}, \mathrm{IF}_{n}\right)$, and $f_{2} \in C\left(I_{c} \times I_{d}, \mathrm{IF}_{n}\right)$ are given functions.

For each $(\lambda, \theta) \in I_{c} \times I_{d}$, we define the state-dependent delays $\left\langle\phi_{1}, \phi_{2}\right\rangle_{(\lambda, \theta)}\left(r_{1}, r_{2}\right)$ by

$$
\left\langle\phi_{1}, \phi_{2}\right\rangle_{(\lambda, \theta)}\left(r_{1}, r_{2}\right)=\left\langle\phi_{1}, \phi_{2}\right\rangle\left(\lambda+r_{1}, \theta+r_{2}\right), \quad\left(r_{1}, r_{2}\right) \in I_{0} .
$$

In the 2nd part of this section, we will prove the existence-uniqueness of intuitionistic fuzzy solution for the following state-dependent delay intuitionistic fuzzy functional partial differential equation of the general model:

$$
\begin{cases}\frac{\partial^{2}\left\langle\phi_{1}, \phi_{2}\right\rangle(\lambda, \theta)}{\partial \lambda \partial \theta}=\frac{\partial\left(q(\lambda, \theta)\left\langle\phi_{1}, \phi_{2}\right\rangle(\lambda, \theta)\right)}{\partial \theta}+G_{2}\left(\lambda, \theta,\left\langle\phi_{1}, \phi_{2}\right\rangle_{(\lambda, \theta)}\right), & (\lambda, \theta) \in I_{c} \times I_{d}:=[0, c] \times[0, d], \\ \left\langle\phi_{1}, \phi_{2}\right\rangle(\lambda, \theta)=\eta(\lambda, \theta), & (\lambda, \theta) \in \widehat{I}_{r}, \\ \left\langle\phi_{1}, \phi_{2}\right\rangle(\lambda, 0)+\int_{0}^{\theta} L_{1}(\lambda)\left\langle\phi_{1}, \phi_{2}\right\rangle(\lambda, \sigma) \mathrm{d} \sigma=f_{1}(\lambda, \theta), & (\lambda, \theta) \in I_{c} \times I_{d}, \\ \left\langle\phi_{1}, \phi_{2}\right\rangle(0, \theta)+\int_{0}^{\lambda} L_{2}(\theta)\left\langle\phi_{1}, \phi_{2}\right\rangle(\zeta, \theta) \mathrm{d} \zeta=f_{2}(\lambda, \theta), & (\lambda, \theta) \in I_{c} \times I_{d},\end{cases}
$$


where $G_{2}: I_{r} \times C\left(I_{0}, \mathrm{IF}_{n}\right) \longrightarrow \mathrm{IF}_{n}, L_{1}, L_{2}, f_{1}$, and $f_{2}$ are as in problem (19), and $q \in C\left(I_{c} \times I_{d}, \mathbb{R}\right)$.

Definition 10. A function $\left\langle\phi_{1}, \phi_{2}\right\rangle \in C\left(I_{r}, \mathrm{IF}_{n}\right)$ can be a solution of model (19) if $\left\langle\phi_{1}, \phi_{2}\right\rangle$ verifies the following equation:

$$
\begin{aligned}
\left\langle\phi_{1}, \phi_{2}\right\rangle(\lambda, \theta)= & P(\lambda, \theta) \int_{0}^{\theta} L_{1}(\lambda)\left\langle\phi_{1}, \phi_{2}\right\rangle(\lambda, \sigma) \mathrm{d} \sigma \\
& -\int_{0}^{\lambda} L_{2}(\theta)\left\langle\phi_{1}, \phi_{2}\right\rangle(\zeta, \theta) \mathrm{d} \zeta \\
& -L_{1}(0) \int_{0}^{\theta} \int_{0}^{\lambda} L_{2}(\sigma)\left\langle\phi_{1}, \phi_{2}\right\rangle(\zeta, \sigma) \mathrm{d} \zeta \mathrm{d} \sigma \\
& +\int_{0}^{\lambda} \int_{0}^{\theta} G_{1}\left(\zeta, \sigma,\left\langle\phi_{1}, \phi_{2}\right\rangle_{(\zeta, \sigma)}\right) \mathrm{d} \sigma \mathrm{d} \zeta
\end{aligned}
$$

if $(\lambda, \theta) \in I_{c} \times I_{d} \quad$ where $P(\lambda, \theta)=f_{1}(\lambda, \theta)+f_{2}(\lambda, \theta)-$ $f_{1}(0, \theta)+L_{1}(0) \int_{0}^{\theta} f_{2}(\lambda, \sigma) \mathrm{d} \sigma$ and $\left\langle\phi_{1}, \phi_{2}\right\rangle(\lambda, \theta)=\eta(\lambda, \theta)$, if $(\lambda, \theta) \in \widehat{I}_{r}$.

Let $L_{1}=\sup _{\lambda \in I_{c}}\left|L_{1}(\lambda)\right|$ and $L_{2}=\sup _{\theta \in I_{d}}\left|L_{2}(\theta)\right|$. By applying Banach fixed point theorem, we will prove the following results.
Theorem 3. Suppose that

(1) $G_{1}: I_{r} \times C\left(I_{0}, I F_{n}\right) \longrightarrow I F_{n}$ is continuous

(2) For $(\lambda, \theta) \in I_{c} \times I_{d}, \quad\left\langle\phi_{1}, \phi_{2}\right\rangle$ and $\left\langle\phi_{1}^{\prime}, \phi_{2}^{\prime}\right\rangle \in C\left(I_{r}\right.$, $\left.I F_{n}\right)$, and $r_{1}, r_{2} \in I_{0}$, we have

$$
\begin{aligned}
& d_{\infty}^{n}\left(G_{1}\left(\lambda, \theta,\left\langle\phi_{1}, \phi_{2}\right\rangle_{(\lambda, \theta)}\right), G_{1}\left(\lambda, \theta,\left\langle\phi_{1}^{\prime}, \phi_{2}^{\prime}\right\rangle_{(\lambda, \theta)}\right)\right) \\
& \leq L d_{\infty}^{n}\left(\left\langle\phi_{1}, \phi_{2}\right\rangle\left(\lambda+r_{1}, \theta+r_{2}\right),\left\langle\phi_{1}^{\prime}, \phi_{2}^{\prime}\right\rangle\left(\lambda+r_{1}, \theta+r_{2}\right)\right),
\end{aligned}
$$

where $L>0$ is a given constant. Moreover, for all $\beta>0$ satisfying

$$
\beta^{2}-\beta\left(L_{1}+L_{2}\right)-L_{1} L_{2}-L>0
$$

Therefore, problem (19) has a unique intuitionistic fuzzy solution in $C\left(I_{r}, \mathrm{IF}_{n}\right)$ with the metric $D_{\beta}$.

Proof. Consider the operator $T_{1}: C\left(I_{r}, \mathrm{IF}_{n}\right) \longrightarrow C\left(I_{r}, \mathrm{IF}_{n}\right)$ defined as follows:

$$
T_{1}\left(\left\langle\phi_{1}, \phi_{2}\right\rangle\right)(\lambda, \theta):=\left\{\begin{array}{l}
\eta(\lambda, \theta),(\lambda, \theta) \in \widehat{I}_{r}, \\
P(\lambda, \theta)-\int_{0}^{\theta} L_{1}(\lambda)\left\langle\phi_{1}, \phi_{2}\right\rangle(\lambda, \sigma) d \sigma-\int_{0}^{\lambda} L_{2}(\theta)\left\langle\phi_{1}, \phi_{2}\right\rangle(\zeta, \theta) d \zeta-L_{1}(0) \int_{0}^{\theta} \int_{0}^{\lambda} L_{2}(\sigma)\left\langle\phi_{1}, \phi_{2}\right\rangle(\zeta, \sigma) d \zeta d \sigma \\
+\int_{0}^{\lambda} \int_{0}^{\theta} G_{1}\left(\zeta, \sigma,\left\langle\phi_{1}, \phi_{2}\right\rangle(\zeta, \sigma)\right) d \sigma d \zeta, \quad \text { if }(\lambda, \theta) \in I_{c} \times I_{d},
\end{array}\right.
$$

where $\quad P(\lambda, \theta)=f_{1}(\lambda, \theta)+\quad f_{2}(\lambda, \theta)-f_{1}(0, \theta)+$ $L_{1}(0) \int_{0}^{\theta} f_{2}(\lambda, \sigma) \mathrm{d} \sigma$.

We will prove that $T_{1}$ is a contraction operator.
Therefore, consider $\left\langle\phi_{1}, \phi_{2}\right\rangle$ and $\left\langle\phi_{1}^{\prime}, \phi_{2}^{\prime}\right\rangle \in C\left(I_{r}, \mathrm{IF}_{n}\right)$, and for $(\lambda, \theta) \in I_{c} \times I_{d}$, we have

$$
\begin{aligned}
T_{1}\left(\left\langle\phi_{1}, \phi_{2}\right\rangle(\lambda, \theta)\right):= & P(\lambda, \theta)-\int_{0}^{\theta} L_{1}(\lambda)\left\langle\phi_{1}, \phi_{2}\right\rangle(\lambda, \sigma) \mathrm{d} \sigma-\int_{0}^{\lambda} L_{2}(\theta)\left\langle\phi_{1}, \phi_{2}\right\rangle(\zeta, \theta) \mathrm{d} \zeta \\
& -L_{1}(0) \int_{0}^{\theta} \int_{0}^{\lambda} L_{2}(\sigma)\left\langle\phi_{1}, \phi_{2}\right\rangle(\zeta, \sigma) \mathrm{d} \zeta \mathrm{d} \sigma+\int_{0}^{\lambda} \int_{0}^{\theta} G_{1}\left(\zeta, \sigma,\left\langle\phi_{1}, \phi_{2}\right\rangle(\zeta, \sigma)\right) \mathrm{d} \sigma \mathrm{d} \zeta, \\
T_{1}\left(\left\langle\phi_{1}^{\prime}, \phi_{2}^{\prime}\right\rangle(\lambda, \theta)\right):= & P(\lambda, \theta)-\int_{0}^{\theta} L_{1}(\lambda)\left\langle\phi_{1}^{\prime}, \phi_{2}^{\prime}\right\rangle(\lambda, \sigma) \mathrm{d} \sigma-\int_{0}^{\lambda} L_{2}(\theta)\left\langle\phi_{1}^{\prime}, \phi_{2}^{\prime}\right\rangle(\zeta, \theta) \mathrm{d} \zeta \\
& -L_{1}(0) \int_{0}^{\theta} \int_{0}^{\lambda} L_{2}(\sigma)\left\langle\phi_{1}^{\prime}, \phi_{2}^{\prime}\right\rangle(\zeta, \sigma) \mathrm{d} \zeta \mathrm{d} \sigma+\int_{0}^{\lambda} \int_{0}^{\theta} G_{1}\left(\zeta, \sigma,\left\langle\phi_{1}^{\prime}, \phi_{2}^{\prime}\right\rangle_{(\zeta, \sigma)}\right) \mathrm{d} \sigma \mathrm{d} \zeta .
\end{aligned}
$$

We have the following inequality: 
Journal of Mathematics

7

$$
\begin{aligned}
d_{\infty}^{n}\left(T_{1}\left(\left\langle\phi_{1}, \phi_{2}\right\rangle\right)(\lambda, \theta), T_{1}\left(\left\langle\phi_{1}^{\prime}, \phi_{2}^{\prime}\right\rangle\right)(\lambda, \theta)\right) \leq & d_{\infty}^{n}\left(\int_{0}^{\theta} L_{1}(\lambda)\left\langle\phi_{1}, \phi_{2}\right\rangle(\lambda, \sigma) \mathrm{d} \sigma, \int_{0}^{\theta} L_{1}(\lambda)\left\langle\phi_{1}^{\prime}, \phi_{2}^{\prime}\right\rangle(\lambda, \sigma) \mathrm{d} \sigma\right) \\
& +d_{\infty}^{n}\left(\int_{0}^{\lambda} L_{2}(\theta)\left\langle\phi_{1}, \phi_{2}\right\rangle(\zeta, \theta) \mathrm{d} \zeta, \int_{0}^{\lambda} L_{2}(\theta)\left\langle\phi_{1}^{\prime}, \phi_{2}^{\prime}\right\rangle(\zeta, \theta) \mathrm{d} \zeta\right) \\
& +d_{\infty}^{n}\left(L_{1}(0) \int_{0}^{\theta} \int_{0}^{\lambda} L_{2}(\sigma)\left\langle\phi_{1}, \phi_{2}\right\rangle(\zeta, \sigma) \mathrm{d} \zeta \mathrm{d} \sigma, L_{1}(0) \int_{0}^{\theta} \int_{0}^{\lambda} L_{2}(\sigma)\left\langle\phi_{1}^{\prime}, \phi_{2}^{\prime}\right\rangle(\zeta, \sigma) \mathrm{d} \zeta \mathrm{d} \sigma\right) \\
& +d_{\infty}^{n}\left(\int_{0}^{\lambda} \int_{0}^{\theta} G_{1}\left(\zeta, \sigma,\left\langle\phi_{1}, \phi_{2}\right\rangle(\zeta, \sigma)\right) \mathrm{d} \sigma \mathrm{d} \zeta, \int_{0}^{\lambda} \int_{0}^{\theta} G_{1}\left(\zeta, \sigma,\left\langle\phi_{1}^{\prime}, \phi_{2}^{\prime}\right\rangle(\zeta, \sigma)\right) \mathrm{d} \sigma \mathrm{d} \zeta\right) \\
\leq & L_{1} \int_{0}^{\theta} d_{\infty}^{n}\left(\left\langle\phi_{1}, \phi_{2}\right\rangle(\lambda, \sigma),\left\langle\phi_{1}^{\prime}, \phi_{2}^{\prime}\right\rangle(\lambda, \sigma)\right) \mathrm{d} \sigma \\
& +L_{2} \int_{0}^{\lambda} d_{\infty}^{n}\left(\left\langle\phi_{1}, \phi_{2}\right\rangle(\zeta, \theta),\left\langle\phi_{1}^{\prime}, \phi_{2}^{\prime}\right\rangle(\zeta, \theta)\right) \mathrm{d} \zeta \\
& +\left|L_{1}(0)\right| \sup _{\theta \in I_{d}}\left|L_{2}(\theta)\right| \int_{0}^{\theta} \int_{0}^{\lambda} d_{\infty}^{n}\left(\left\langle\phi_{1}, \phi_{2}\right\rangle(\zeta, \sigma),\left\langle\phi_{1}^{\prime}, \phi_{2}^{\prime}\right\rangle(\zeta, \sigma)\right) \mathrm{d} \zeta \mathrm{d} \sigma \\
& +\int_{0}^{\lambda} \int_{0}^{\theta} d_{\infty}^{n}\left(G_{1}\left(\zeta, \sigma,\left\langle\phi_{1}, \phi_{2}\right\rangle{ }_{(\zeta, \sigma)}\right), G_{1}\left(\zeta, \sigma,\left\langle\phi_{1}^{\prime}, \phi_{2}^{\prime}\right\rangle(\zeta, \sigma)\right) \mathrm{d} \sigma \mathrm{d} \zeta .\right.
\end{aligned}
$$

Multiplying (27) by $e^{-\beta(\lambda+\theta)}$, then we get

$$
\begin{aligned}
& e^{-\beta(\lambda+\theta)} d_{\infty}^{n}\left(\int_{0}^{\lambda} \int_{0}^{\theta} G_{1}\left(\zeta, \sigma,\left\langle\phi_{1}, \phi_{2}\right\rangle\left(\zeta+r_{1}, \sigma+r_{2}\right)\right), \int_{0}^{\lambda} \int_{0}^{\theta} G_{1}\left(\zeta, \sigma,\left\langle\phi_{1}^{\prime}, \phi_{2}^{\prime}\right\rangle\left(\zeta+r_{1}, \sigma+r_{2}\right)\right)\right) \mathrm{d} \sigma \mathrm{d} \zeta e^{-\beta(\lambda+\theta)} \\
& \quad \leq L \int_{0}^{\lambda} \int_{0}^{\theta} d_{\infty}^{n}\left(\left\langle\phi_{1}, \phi_{2}\right\rangle\left(\zeta+r_{1}, \sigma+r_{2}\right),\left\langle\phi_{1}^{\prime}, \phi_{2}^{\prime}\right\rangle\left(\zeta+r_{1}, \sigma+r_{2}\right)\right) \mathrm{d} \sigma \mathrm{d} \zeta e^{-\beta(\lambda+\theta)} \\
& \quad \leq L D_{\beta}\left(\left\langle\phi_{1}, \phi_{2}\right\rangle,\left\langle\phi_{1}^{\prime}, \phi_{2}^{\prime}\right\rangle\right) \int_{0}^{\lambda} \int_{0}^{\theta} e^{\beta\left(r_{1}+r_{2}+\zeta+\sigma\right)} \mathrm{d} \sigma \mathrm{d} \zeta e^{-\beta(\lambda+\theta)} \\
& \quad \leq L D_{\beta}\left(\left\langle\phi_{1}, \phi_{2}\right\rangle,\left\langle\phi_{1}^{\prime}, \phi_{2}^{\prime}\right\rangle\right) \frac{1}{\beta} e^{\beta\left(r_{1}+r_{2}\right)} \int_{0}^{\lambda}\left(e^{\beta(\zeta+\theta)}-e^{\beta \zeta}\right) \mathrm{d} \zeta e^{-\beta(\lambda+\theta)} \\
& \quad \leq \frac{L}{\beta^{2}} D_{\beta}\left(\left\langle\phi_{1}, \phi_{2}\right\rangle,\left\langle\phi_{1}^{\prime}, \phi_{2}^{\prime}\right\rangle\right) e^{\beta\left(r_{1}+r_{2}\right)}\left(e^{\beta \lambda}-1\right)\left(e^{\beta \theta}-1\right) e^{-\beta(\lambda+\theta)} \\
& \quad \leq \frac{L}{\beta^{2}} D_{\beta}\left(\left\langle\phi_{1}, \phi_{2}\right\rangle,\left\langle\phi_{1}^{\prime}, \phi_{2}^{\prime}\right\rangle\right) .
\end{aligned}
$$

The same, we have

$$
\begin{aligned}
e^{-\beta(\lambda+\theta)}\left|L_{1}(0)\right| \sup _{y \in I_{d}}\left|L_{2}(\theta)\right| \int_{0}^{\theta} \int_{0}^{\lambda} d_{\infty}^{n}\left(\left\langle\phi_{1}, \phi_{2}\right\rangle(\zeta, \sigma),\left\langle\phi_{1}^{\prime}, \phi_{2}^{\prime}\right\rangle(\zeta, \sigma)\right) \mathrm{d} \zeta \mathrm{d} \sigma \leq \frac{L_{1} L_{2}}{\beta^{2}} D_{\beta}\left(\left\langle\phi_{1}, \phi_{2}\right\rangle,\left\langle\phi_{1}^{\prime}, \phi_{2}^{\prime}\right\rangle\right), \\
e^{-\beta(\lambda+\theta)} L_{1} \int_{0}^{\theta} d_{\infty}^{n}\left(\left\langle\phi_{1}, \phi_{2}\right\rangle(\lambda, \sigma),\left\langle\phi_{1}^{\prime}, \phi_{2}^{\prime}\right\rangle(\lambda, \sigma)\right) \mathrm{d} \sigma \leq L_{1} D_{\beta}\left(\left\langle\phi_{1}, \phi_{2}\right\rangle,\left\langle\phi_{1}^{\prime}, \phi_{2}^{\prime}\right\rangle\right) \int_{0}^{\theta} e^{\beta(\lambda+\sigma)} \mathrm{d} \sigma e^{-\beta(\lambda+\theta)} \\
\leq L_{1} D_{\beta}\left(\left\langle\phi_{1}, \phi_{2}\right\rangle,\left\langle\phi_{1}^{\prime}, \phi_{2}^{\prime}\right\rangle\right)\left(e^{-\beta(\lambda+\theta)}-e^{\beta \theta}\right) e^{-\beta(\lambda+\theta)} \\
\leq \frac{L_{1}}{\beta} D_{\beta}\left(\left\langle\phi_{1}, \phi_{2}\right\rangle,\left\langle\phi_{1}^{\prime}, \phi_{2}^{\prime}\right\rangle\right) .
\end{aligned}
$$


Similarly, we get

$$
\begin{aligned}
& e^{-\beta(\lambda+\theta)} L_{2} \int_{0}^{\lambda} d_{\infty}^{n}\left(\left\langle\phi_{1}, \phi_{2}\right\rangle(\zeta, \theta),\left\langle\phi_{1}^{\prime}, \phi_{2}^{\prime}\right\rangle(\zeta, \theta)\right) \mathrm{d} \zeta \\
& \quad \leq \frac{L_{2}}{\beta} D_{\beta}\left(\left\langle\phi_{1}, \phi_{2}\right\rangle,\left\langle\phi_{1}^{\prime}, \phi_{2}^{\prime}\right\rangle\right) .
\end{aligned}
$$

From (28)-(31), we obtain

$$
\begin{gathered}
e^{-\beta(\lambda+\theta)} d_{\infty}^{n}\left(T_{1}\left(\left\langle\phi_{1}, \phi_{2}\right\rangle\right)(\lambda, \theta), T_{1}\left(\left\langle\phi_{1}^{\prime}, \phi_{2}^{\prime}\right\rangle\right)(\lambda, \theta)\right) \\
\leq\left(\frac{L_{1}+L_{2}}{\beta}+\frac{L_{1} L_{2}+L}{\beta^{2}}\right) D_{\beta}\left(\left\langle\phi_{1}, \phi_{2}\right\rangle,\left\langle\phi_{1}^{\prime}, \phi_{2}^{\prime}\right\rangle\right) .
\end{gathered}
$$

If $(\lambda, \theta) \in \widehat{I}_{r}$,

$d_{\infty}^{n}\left(T_{1}\left(\left\langle\phi_{1}, \phi_{2}\right\rangle\right)(\lambda, \theta), T_{1}\left(\left\langle\phi_{1}^{\prime}, \phi_{2}^{\prime}\right\rangle\right)(\lambda, \theta)\right)=0$.
Hence, for each $(\lambda, \theta) \in I_{r}$,

$$
\begin{aligned}
& D_{\beta}\left(T_{1}\left(\left\langle\phi_{1}, \phi_{2}\right\rangle\right), T_{1}\left(\left\langle\phi_{1}^{\prime}, \phi_{2}^{\prime}\right\rangle\right)\right) \\
& \quad \leq\left(\frac{L_{1}+L_{2}}{\beta}+\frac{L_{1} L_{2}+L}{\beta^{2}}\right) D_{\beta}\left(\left\langle\phi_{1}, \phi_{2}\right\rangle,\left\langle\phi_{1}^{\prime}, \phi_{2}^{\prime}\right\rangle\right) .
\end{aligned}
$$

So, for all $\beta>0$ satisfying (31), we have $\left(\left(\left(L_{1}+L_{2}\right) / \beta\right)+\left(\left(L_{1} L_{2}+L\right) / \beta^{2}\right)\right)<1$. Then, $T_{1}$ is a contraction operator.

Therefore, $T_{1}$ has a unique fixed point, which is the solution of integral boundary problem (21).

Definition 11. A function $\left\langle\phi_{1}, \phi_{2}\right\rangle \in C\left(I_{r}, \mathrm{IF}_{n}\right)$ is called an intuitionistic fuzzy solution of problem (21) if $\left\langle\phi_{1}, \phi_{2}\right\rangle$ verifies the following integral equation:

$$
\begin{aligned}
& \left\langle\phi_{1}, \phi_{2}\right\rangle(\lambda, \theta)=P(\lambda, \theta)-\int_{0}^{\theta} L_{1}(\lambda)\left\langle\phi_{1}, \phi_{2}\right\rangle(\lambda, \sigma) \mathrm{d} \sigma-\int_{0}^{\lambda} L_{2}(\theta)\left\langle\phi_{1}, \phi_{2}\right\rangle(\zeta, \theta) \mathrm{d} \zeta \\
& +\int_{0}^{\lambda} q(\zeta, \theta)\left\langle\phi_{1}, \phi_{2}\right\rangle(\zeta, \theta) \mathrm{d} \zeta-L_{1}(0) \int_{0}^{\theta} \int_{0}^{\lambda} L_{2}(\sigma)\left\langle\phi_{1}, \phi_{2}\right\rangle(\zeta, \sigma) \mathrm{d} \zeta \mathrm{d} \sigma \\
& +\int_{0}^{\lambda} q(\zeta, 0)\left\langle\phi_{1}, \phi_{2}\right\rangle(\zeta, 0) \mathrm{d} \zeta+\int_{0}^{\lambda} \int_{0}^{\theta} G_{2}\left(\zeta, \sigma,\left\langle\phi_{1}, \phi_{2}\right\rangle_{(\zeta, \sigma)}\right) \mathrm{d} \sigma \mathrm{d} \zeta, \\
& d_{\infty}^{n}\left(G_{2}\left(\lambda, \theta,\left\langle\phi_{1}, \phi_{2}\right\rangle_{(\lambda, \theta)}\right), G_{2}\left(\lambda, \theta,\left\langle\phi_{1}^{\prime}, \phi_{2}^{\prime}\right\rangle_{(\lambda, \theta)}\right)\right) \\
& \leq L d^{n} \infty\left(\left\langle\phi_{1}, \phi_{2}\right\rangle(\lambda+r 1, \theta+r 2),\left\langle\phi_{1}^{\prime}, \phi_{2}^{\prime}\right\rangle(\lambda+r 1, \theta+r 2)\right),
\end{aligned}
$$

if $(\lambda, \theta) \in I_{c} \times I_{d_{\theta}} \quad$ where $P(\lambda, \theta)=f_{1}(\lambda, \theta)+f_{2}(\lambda, \theta)-$ $f_{1}(0, \theta)+L_{1}(0) \int_{0}^{\theta} f_{2}(\lambda, \sigma) \mathrm{d} \sigma$ and $\left\langle\phi_{1}, \phi_{2}\right\rangle(\lambda, \theta)=\eta(\lambda, \theta)$ if $(\lambda, \theta) \in \widehat{I}_{r}$.

Let $\quad L_{1}=\sup _{\lambda \in I_{c}}\left|L_{1}(\lambda)\right|, \quad L_{2}=\sup _{\theta \in I_{d}}\left|L_{2}(\theta)\right|, \quad$ and $q=\sup _{(\lambda, \theta) \in I_{c} \times I_{d}}|q(\lambda, \theta)|$. By applying the Banach fixed point theorem, we will prove the following result.

Theorem 4. Suppose that

(1) A map $G_{2}: I_{r} \times C\left(I_{0}, I F_{n}\right) \longrightarrow I F_{n}$ is continuous

(2) For $\quad(\lambda, \theta) \in I_{c} \times I_{d}, \quad\left\langle\phi_{1}, \phi_{2}\right\rangle$ and $\left\langle\phi_{1}^{\prime}, \phi_{2}^{\prime}\right\rangle \in$ $C\left(I_{r}, I F_{n}\right)$, and $r_{1}, r_{2} \in I_{0}$, we have where $L>0$ is a given constant. Then, for all $\beta>0$ satisfying

$$
\beta^{2}-\beta\left(L_{1}+L_{2}+2 q\right)-L_{1} L_{2}-L>0 .
$$

Therefore, problem (21) has a unique intuitionistic fuzzy solution in $C\left(I_{r}, \mathrm{IF}_{n}\right)$ with the metric $D_{\beta}$.

Proof. To prove the existence-uniqueness result, we will show that $T_{2}$ has a unique fixed point.

Define operator $T_{2}: C\left(I_{r}, \mathrm{IF}_{n}\right) \longrightarrow C\left(I_{r}, \mathrm{IF}_{n}\right)$ by

$$
T_{2}\left(\left\langle\phi_{1}, \phi_{2}\right\rangle\right)(\lambda, \theta):= \begin{cases}\eta(\lambda, \theta), & (\lambda, \theta) \in \widehat{I}_{r}, \\ P(\lambda, \theta)-\int_{0}^{\theta} L_{1}(\lambda)\left\langle\phi_{1}, \phi_{2}\right\rangle(\lambda, \sigma) \mathrm{d} \sigma-\int_{0}^{\lambda} L_{2}(\theta)\left\langle\phi_{1}, \phi_{2}\right\rangle(\zeta, \theta) \mathrm{d} \zeta+\int_{0}^{\lambda} q(\zeta, \theta)\left\langle\phi_{1}, \phi_{2}\right\rangle(\zeta, \theta) \mathrm{d} \zeta & \text { if }(\lambda, \theta) \in I_{c} \times I_{d}, \\ -L_{1}(0) \int_{0}^{\theta} \int_{0}^{\lambda} L_{2}(\sigma)\left\langle\phi_{1}, \phi_{2}\right\rangle(\zeta, \sigma) \mathrm{d} \zeta \mathrm{d} \sigma+\int_{0}^{\lambda} q(\zeta, 0)\left\langle\phi_{1}, \phi_{2}\right\rangle(\zeta, 0) \mathrm{d} \zeta & \\ + & \int_{0}^{\lambda} \int_{0}^{\theta} G_{2}\left(\zeta, \sigma,\left\langle\phi_{1}, \phi_{2}\right\rangle_{(\zeta, \sigma)}\right) \mathrm{d} \sigma \mathrm{d} \zeta\end{cases}
$$

where 


$$
P(\lambda, \theta)=f_{1}(\lambda, \theta)+f_{2}(\lambda, \theta)-f_{1}(0, \theta)+L_{1}(0) \int_{0}^{\theta} f_{2}(\lambda, \sigma) \mathrm{d} \sigma .
$$

We prove that $T_{2}$ is a contraction operator. Then, consider $\left\langle\phi_{1}, \phi_{2}\right\rangle$ and $\left\langle\phi_{1}^{\prime}, \phi_{2}^{\prime}\right\rangle \in C\left(I_{r}, \mathrm{IF}_{n}\right)$.

We can see that $d_{\infty}^{n}\left(T_{2}\left(\left\langle\phi_{1}, \phi_{2}\right\rangle\right)\right.$ $\left.(\lambda, \theta), T_{2}\left(\left\langle\phi_{1}^{\prime}, \phi_{2}^{\prime}\right\rangle\right)(\lambda, \theta)\right)=0$ if $(\lambda, \theta) \in I_{0}$. On the contrary, on $(\lambda, \theta) \in I_{c} \times I_{d}$, we have

$$
\begin{aligned}
T_{2}\left(\left\langle\phi_{1}, \phi_{2}\right\rangle(\lambda, \theta)\right):= & P(\lambda, \theta)-\int_{0}^{\theta} L_{1}(\lambda)\left\langle\phi_{1}, \phi_{2}\right\rangle(\lambda, \sigma) \mathrm{d} \sigma-\int_{0}^{\lambda} L_{2}(\theta)\left\langle\phi_{1}, \phi_{2}\right\rangle(\zeta, \theta) \mathrm{d} \zeta+\int_{0}^{\lambda} q(\zeta, \theta)\left\langle\phi_{1}, \phi_{2}\right\rangle(\zeta, \theta) \mathrm{d} \zeta \\
& -L_{1}(0) \int_{0}^{\theta} \int_{0}^{\lambda} L_{2}(\sigma)\left\langle\phi_{1}, \phi_{2}\right\rangle(\zeta, \sigma) \mathrm{d} \zeta \mathrm{d} \sigma+\int_{0}^{\lambda} q(\zeta, 0)\left\langle\phi_{1}, \phi_{2}\right\rangle(\zeta, 0) \mathrm{d} \zeta+\int_{0}^{\lambda} \int_{0}^{\theta} G_{2}\left(\zeta, \sigma,\left\langle\phi_{1}, \phi_{2}\right\rangle(\zeta, \sigma)\right) \mathrm{d} \sigma \mathrm{d} \zeta, \\
T_{2}\left(\left\langle\phi_{1}^{\prime}, \phi_{2}^{\prime}\right\rangle(\lambda, \theta)\right):= & P(\lambda, \theta)-\int_{0}^{\theta} L_{1}(\lambda)\left\langle\phi_{1}^{\prime}, \phi_{2}^{\prime}\right\rangle(\lambda, \sigma) \mathrm{d} \sigma-\int_{0}^{\lambda} L_{2}(\theta)\left\langle v^{\prime}, w^{\prime}\right\rangle(\zeta, \theta) \mathrm{d} \zeta+\int_{0}^{\lambda} q(\zeta, \theta)\left\langle\phi_{1}^{\prime}, \phi_{2}^{\prime}\right\rangle(\zeta, \theta) \mathrm{d} \zeta \\
& -L_{1}(0) \int_{0}^{\theta} \int_{0}^{\lambda} L_{2}(\sigma)\left\langle\phi_{1}^{\prime}, \phi_{2}^{\prime}\right\rangle(\zeta, \sigma) \mathrm{d} \zeta \mathrm{d} \sigma+\int_{0}^{\lambda} q(\zeta, 0)\left\langle\phi_{1}^{\prime}, \phi_{2}^{\prime}\right\rangle(\zeta, 0) \mathrm{d} \zeta+\int_{0}^{\lambda} \int_{0}^{\theta} G_{2}\left(\zeta, \sigma,\left\langle\phi_{1}^{\prime}, \phi_{2}^{\prime}\right\rangle(\zeta, \sigma)\right) \mathrm{d} \sigma \mathrm{d} \zeta
\end{aligned}
$$

From the properties of supremum metric, we have

$$
\begin{aligned}
d_{\infty}^{n}\left(T_{2}\left(\left\langle\phi_{1}, \phi_{2}\right\rangle\right)(\lambda, \theta), T_{2}\left(\left\langle\phi_{1}^{\prime}, \phi_{2}^{\prime}\right\rangle\right)(\lambda, \theta)\right) \leq & d_{\infty}^{n}\left(\int_{0}^{\theta} L_{1}(\lambda)\left\langle\phi_{1}, \phi_{2}\right\rangle(\lambda, \sigma) \mathrm{d} \sigma, \int_{0}^{\theta} L_{1}(\lambda)\left\langle\phi_{1}^{\prime}, \phi_{2}^{\prime}\right\rangle(\lambda, \sigma) \mathrm{d} \sigma\right) \\
& +d_{\infty}^{n}\left(\int_{0}^{\lambda} L_{2}(\theta)\left\langle\phi_{1}, \phi_{2}\right\rangle(\zeta, \theta) \mathrm{d} \zeta, \int_{0}^{\lambda} L_{2}(\theta)\left\langle\phi_{1}^{\prime}, \phi_{2}^{\prime}\right\rangle(\zeta, \theta) \mathrm{d} \zeta\right) \\
& +d_{\infty}^{n}\left(L_{1}(0) \int_{0}^{\theta} \int_{0}^{\lambda} L_{2}(\sigma)\left\langle\phi_{1}, \phi_{2}\right\rangle(\zeta, \sigma) \mathrm{d} \zeta \mathrm{d} \sigma, L_{1}(0) \int_{0}^{\theta} \int_{0}^{\lambda} L_{2}(\sigma)\left\langle\phi_{1}^{\prime}, \phi_{2}^{\prime}\right\rangle(\zeta, \sigma) \mathrm{d} \zeta \mathrm{d} \sigma\right) \\
& +d_{\infty}^{n}\left(\int_{0}^{\lambda} q(\zeta, \theta)\left\langle\phi_{1}, \phi_{2}\right\rangle(\zeta, \theta) \mathrm{d} \zeta, \int_{0}^{\lambda} q(\zeta, \theta)\left\langle\phi_{1}^{\prime}, \phi_{2}^{\prime}\right\rangle(\zeta, \theta) \mathrm{d} \zeta\right) \\
& +d_{\infty}^{n}\left(\int_{0}^{\lambda} q(\zeta, 0)\left\langle\phi_{1}, \phi_{2}\right\rangle(\zeta, 0) \mathrm{d} \zeta, \int_{0}^{\lambda} q(\zeta, 0)\left\langle\phi_{1}^{\prime}, \phi_{2}^{\prime}\right\rangle(\zeta, 0) \mathrm{d} \zeta\right) \\
& +d_{\infty}^{n}\left(\int_{0}^{\lambda} \int_{0}^{\theta} G_{2}\left(\zeta, \sigma,\left\langle\phi_{1}, \phi_{2}\right\rangle(\zeta, \sigma)\right) \mathrm{d} \sigma \mathrm{d} \zeta, \int_{0}^{\lambda} \int_{0}^{\theta} G_{2}\left(\zeta, \sigma,\left\langle\phi_{1}^{\prime}, \phi_{2}^{\prime}\right\rangle(\zeta, \sigma)\right) \mathrm{d} \sigma \mathrm{d} \zeta\right), \\
\leq & L_{1} \int_{0}^{\theta} d_{\infty}^{n}\left(\left\langle\phi_{1}, \phi_{2}\right\rangle(\lambda, \sigma),\left\langle\phi_{1}^{\prime}, \phi_{2}^{\prime}\right\rangle(\lambda, \sigma)\right) \mathrm{d} \sigma \\
& +L_{2} \int_{0}^{\lambda} d_{\infty}^{n}\left(\left\langle\phi_{1}, \phi_{2}\right\rangle(\zeta, \theta),\left\langle\phi_{1}^{\prime}, \phi_{2}^{\prime}\right\rangle(\zeta, \theta)\right) \mathrm{d} \zeta \\
& +\left|L_{1}(0)\right| \sup _{\theta \in I_{d}}\left|L_{2}(\theta)\right| \int_{0}^{\theta} \int_{0}^{\lambda} d_{\infty}^{n}\left(\left\langle\phi_{1}, \phi_{2}\right\rangle(\zeta, \sigma),\left\langle\phi_{1}^{\prime}, \phi_{2}^{\prime}\right\rangle(\zeta, \sigma)\right) \mathrm{d} \zeta \mathrm{d} \sigma \\
& +\sup _{(\zeta, \sigma) \in I_{c} \times I_{d}}|q(\zeta, \sigma)| \int_{0}^{\lambda} d_{\infty}^{n}\left(\left\langle\phi_{1}, \phi_{2}\right\rangle(\zeta, \theta),\left\langle\phi_{1}^{\prime}, \phi_{2}^{\prime}\right\rangle(\zeta, \theta)\right) \mathrm{d} \zeta \\
& +\sup _{(\zeta, \sigma) \in I_{c} \times I_{d}}^{\lambda}|q(\zeta, 0)| \int_{0}^{\theta} d_{\infty}^{n}\left(\left\langle\phi_{1}, \phi_{2}\right\rangle(\zeta, 0),\left\langle\phi_{1}^{\prime}, \phi_{2}^{\prime}\right\rangle(\zeta, 0)\right) \mathrm{d} \zeta \\
& \left.+G_{2}\left(\zeta, \sigma,\left\langle\phi_{1}, \phi_{2}\right\rangle\right\rangle_{(\zeta, \sigma)}\right), G_{2}\left(\zeta, \sigma,\left\langle\phi_{1}^{\prime}, \phi_{2}^{\prime}\right\rangle(\zeta, 0)\right) \mathrm{d} \sigma \mathrm{d} \zeta .
\end{aligned}
$$


Set $\quad L_{1}=\sup _{\lambda \in I_{c}}\left|L_{1}(\lambda)\right|, \quad L_{2}=\sup _{\theta \in I_{d}}\left|L_{2}(\theta)\right|, \quad$ and $q=\sup _{(\lambda, \theta) \in I_{c} \times I_{d}}|q(\lambda, \theta)|$. We get the following assessment:

$$
\begin{aligned}
& \sup _{(\zeta, \sigma) \in I_{c} \times I_{d}}|q(\zeta, \sigma)| \int_{0}^{\lambda} d_{\infty}^{n}\left(\left\langle\phi_{1}, \phi_{2}\right\rangle(\zeta, \theta),\left\langle\phi_{1}^{\prime}, \phi_{2}^{\prime}\right\rangle(\zeta, \theta)\right) \mathrm{d} \zeta \\
& \quad=q \int_{0}^{\lambda} d_{\infty}^{n}\left(\left\langle\phi_{1}, \phi_{2}\right\rangle(\zeta, \theta),\left\langle\phi_{1}^{\prime}, \phi_{2}^{\prime}\right\rangle(\zeta, \theta)\right) \mathrm{d} \zeta \\
& \leq q D_{\beta}\left(\left\langle\phi_{1}, \phi_{2}\right\rangle,\left\langle\phi_{1}^{\prime}, \phi_{2}^{\prime}\right\rangle\right) \int_{0}^{\lambda} e^{\beta(\zeta+\theta)} \mathrm{d} \zeta \\
& \quad \leq q D_{\beta}\left(\left\langle\phi_{1}, \phi_{2}\right\rangle,\left\langle\phi_{1}^{\prime}, \phi_{2}^{\prime}\right\rangle\right) \frac{1}{\beta}\left(e^{\beta(\zeta+\theta)}-e^{\beta \theta}\right) .
\end{aligned}
$$

Multiplying (42) by $\exp ^{-\beta(\lambda+\theta)}$, we get

$$
\begin{aligned}
& e^{-\beta(\lambda+\theta)} \sup _{(\zeta, \sigma) \in I_{c} \times I_{d}}|q(\zeta, \sigma)| \int_{0}^{\lambda} d_{\infty}^{n}\left(\left\langle\phi_{1}, \phi_{2}\right\rangle(\zeta, \theta),\left\langle\phi_{1}^{\prime}, \phi_{2}^{\prime}\right\rangle(\zeta, \theta)\right) \mathrm{d} \zeta \\
& \leq q D_{\beta}\left(\left\langle\phi_{1}, \phi_{2}\right\rangle,\left\langle\phi_{1}^{\prime}, \phi_{2}^{\prime}\right\rangle\right) \frac{1}{\beta}\left(e^{\beta(\lambda+\theta)}-e^{\beta \theta}\right) e^{-\beta(\lambda+\theta)}, \\
& \leq \frac{q}{\beta} D_{\beta}\left(\left\langle\phi_{1}, \phi_{2}\right\rangle,\left\langle\phi_{1}^{\prime}, \phi_{2}^{\prime}\right\rangle\right) .
\end{aligned}
$$

Similarly, we obtain

$$
\begin{aligned}
& e^{-\beta(\lambda+\theta)} \sup _{(\zeta, 0) \in I_{c} \times I_{d}}|q(\zeta, 0)| \int_{0}^{\lambda} d_{\infty}^{n}\left(\left\langle\phi_{1}, \phi_{2}\right\rangle(\zeta, 0),\left\langle\phi_{1}^{\prime}, \phi_{2}^{\prime}\right\rangle(\zeta, 0)\right) \mathrm{d} \sigma \\
& \leq \frac{q}{\beta} D_{\beta}\left(\left\langle\phi_{1}, \phi_{2}\right\rangle,\left\langle\phi_{1}^{\prime}, \phi_{2}^{\prime}\right\rangle\right) .
\end{aligned}
$$

From (30) and (31), we imply

$$
\begin{aligned}
& e^{-\beta(\lambda+\theta)} L_{1} \int_{0}^{\theta} d_{\infty}^{n}\left(\left\langle\phi_{1}, \phi_{2}\right\rangle(\lambda, \sigma),\left\langle\phi_{1}^{\prime}, \phi_{2}^{\prime}\right\rangle(\lambda, \sigma)\right) \mathrm{d} \sigma \\
& \leq \frac{L_{1}}{\beta} D_{\beta}\left(\left\langle\phi_{1}, \phi_{2}\right\rangle,\left\langle\phi_{1}^{\prime}, \phi_{2}^{\prime}\right\rangle\right), \\
& e^{-\beta(\lambda+\theta)} L_{2} \int_{0}^{\lambda} d_{\infty}^{n}\left(\left\langle\phi_{1}, \phi_{2}\right\rangle(\zeta, \theta),\left\langle\phi_{1}^{\prime}, \phi_{2}^{\prime}\right\rangle(\zeta, \theta)\right) \mathrm{d} \zeta \\
& \leq \frac{L_{2}}{\beta} D_{\beta}\left(\left\langle\phi_{1}, \phi_{2}\right\rangle,\left\langle\phi_{1}^{\prime}, \phi_{2}^{\prime}\right\rangle\right) .
\end{aligned}
$$

and from (28), we also get

$$
\begin{aligned}
& e^{-\beta(\lambda+\theta)} d_{\infty}^{n}\left(\int_{0}^{\lambda} \int_{0}^{\theta} G_{2}\left(\zeta, \sigma,\left\langle\phi_{1}, \phi_{2}\right\rangle\left(\zeta+r_{1}, \sigma+r_{2}\right)\right), \int_{0}^{\lambda} \int_{0}^{\theta} G_{2}\left(\zeta, \sigma,\left\langle\phi_{1}^{\prime}, \phi_{2}^{\prime}\right\rangle\left(\zeta+r_{1}, \sigma+r_{2}\right)\right)\right) \mathrm{d} \sigma \mathrm{d} \zeta e^{-\beta(\lambda+\theta)} \\
& \quad \leq \frac{L}{\beta^{2}} D_{\beta}\left(\left\langle\phi_{1}, \phi_{2}\right\rangle,\left\langle\phi_{1}^{\prime}, \phi_{2}^{\prime}\right\rangle\right) \\
& e^{-\beta(\lambda+\theta)}\left|L_{1}(0)\right| \sup _{y \in I_{d}}\left|L_{2}(\theta)\right| \int_{0}^{\theta} \int_{0}^{\lambda} d_{\infty}^{n}\left(\left\langle\phi_{1}, \phi_{2}\right\rangle(\zeta, \sigma),\left\langle\phi_{1}^{\prime}, \phi_{2}^{\prime}\right\rangle(\zeta, \sigma)\right) \mathrm{d} \zeta \mathrm{d} \sigma \leq \frac{L_{1} L_{2}}{\beta^{2}} D_{\beta}\left(\left\langle\phi_{1}, \phi_{2}\right\rangle,\left\langle\phi_{1}^{\prime}, \phi_{2}^{\prime}\right\rangle\right)
\end{aligned}
$$

From (43)-(48), we have

$$
\begin{aligned}
& e^{-\beta(\lambda+\theta)} d_{\infty}^{n}\left(T_{2}\left(\left\langle\phi_{1}, \phi_{2}\right\rangle\right)(\lambda, \theta), T_{2}\left(\left\langle\phi_{1}^{\prime}, \phi_{2}^{\prime}\right\rangle\right)(\lambda, \theta)\right) \\
& \quad \leq\left(\frac{L_{1}+L_{2}+2 q}{\beta}+\frac{L_{1} L_{2}+L}{\beta^{2}}\right) D_{\beta}\left(\left\langle\phi_{1}, \phi_{2}\right\rangle,\left\langle\phi_{1}^{\prime}, \phi_{2}^{\prime}\right\rangle\right) .
\end{aligned}
$$

If $(\lambda, \theta) \in \widehat{I}_{r}$,

$$
d_{\infty}^{n}\left(T_{2}\left(\left\langle\phi_{1}, \phi_{2}\right\rangle\right)(\lambda, \theta), T_{2}\left(\left\langle\phi_{1}^{\prime}, \phi_{2}^{\prime}\right\rangle\right)(\lambda, \theta)\right)=0 .
$$

Hence, for each $(\lambda, \theta) \in I_{r}$,

$$
\begin{aligned}
& D_{\beta}\left(T_{2}\left(\left\langle\phi_{1}, \phi_{2}\right\rangle\right), T_{2}\left(\left\langle\phi_{1}^{\prime}, \phi_{2}^{\prime}\right\rangle\right)\right) \\
& \quad \leq\left(\frac{L_{1}+L_{2}+2 q}{\beta}+\frac{L_{1} L_{2}+L}{\beta^{2}}\right) D_{\beta}\left(\left\langle\phi_{1}, \phi_{2}\right\rangle,\left\langle\phi_{1}^{\prime}, \phi_{2}^{\prime}\right\rangle\right) .
\end{aligned}
$$

Then, for all $\beta>0$ verifying (36), it implies $\left(\left(L_{1}+\right.\right.$ $\left.\left.\left.L_{2}+2 q\right) / \beta\right)+\left(\left(L_{1} L_{2}+L\right) / \beta^{2}\right)\right)<1$; therefore, $T_{2}$ is a contraction operator.

Thus, $T_{2}$ has a unique fixed point, which is the solution of (21).

\section{Examples}

We propose the following two examples: to illustrate the usefulness of our main results and to find the intuitionistic fuzzy solution, we will use the method of steps proposed in $[22,23]$.

Example 1. Now, we present a first example to illustrate Theorem 3; consider the following state-dependent delay intuitionistic fuzzy partial functional differential equations for some $w>0$ in the following form: 


$$
\begin{array}{r}
\frac{\partial^{2}\left\langle\phi_{1}, \phi_{2}\right\rangle(\lambda, \theta)}{\partial \lambda \partial \theta}=w^{2} e^{w / 2}\left\langle\phi_{1}, \phi_{2}\right\rangle\left(\lambda-\frac{1}{4}, \theta-\frac{1}{4}\right), \\
(\lambda, \theta) \in[0,1] \times[0,1] .
\end{array}
$$

And, the integral boundary initial conditions are

$$
\left\langle\phi_{1}, \phi_{2}\right\rangle(\lambda, 0)+\frac{1}{8} \int_{0}^{\theta}\left\langle\phi_{1}, \phi_{2}\right\rangle(\lambda, \sigma) \mathrm{d} \sigma=\frac{C}{8 w} e^{w \lambda}\left(e^{w \theta}+8 w-1\right),
$$

$$
\left\langle\phi_{1}, \phi_{2}\right\rangle(0, \theta)+\frac{1}{8} \int_{0}^{\lambda}\left\langle\phi_{1}, \phi_{2}\right\rangle(\zeta, \theta) \mathrm{d} \zeta=\frac{C}{8 w} e^{w \theta}\left(e^{w \lambda}+8 w-1\right),
$$

where $C \in \mathrm{IF}_{1}$ is a triangular intuitionistic fuzzy number, and the initial condition is

$$
\left\langle\phi_{1}, \phi_{2}\right\rangle(\lambda, \theta)=C\left(e^{w \lambda}+e^{w \theta}+\lambda \theta\right),
$$

for $(\lambda, \theta t \in n[-(1 / 4), 1] q \times h[-(1 / 4), 1], x(0,1] 7 \times C(0,1]$.

Since

$$
G_{1}\left(\lambda, \theta,\left\langle\phi_{1}, \phi_{2}\right\rangle_{(\lambda, \theta)}\right)=w^{2} e^{w / 2}\left\langle\phi_{1}, \phi_{2}\right\rangle\left(\lambda-\frac{1}{4}, \theta-\frac{1}{4}\right),
$$

it satisfies hypothesises (1) and (2) of Theorem 3.

Indeed, it is easy to see that $G_{1}$ is continuous and

$$
\begin{aligned}
& d_{\infty}\left(G_{1}\left(\lambda, \theta,\left\langle\phi_{1}, \phi_{2}\right\rangle_{(\lambda, \theta)}\right), G_{1}\left(\lambda, \theta,\left\langle\phi_{1}^{\prime}, \phi_{2}^{\prime}\right\rangle_{(\lambda, \theta)}\right)\right) \\
& \quad \leq w^{2} e^{w / 2} d_{\infty}\left(\left\langle\phi_{1}, \phi_{2}\right\rangle_{(\lambda, \theta)}\left(-\frac{1}{4},-\frac{1}{4}\right),\left\langle\phi_{1}^{\prime}, \phi_{2}^{\prime}\right\rangle_{(\lambda, \theta)}\left(-\frac{1}{4},-\frac{1}{4}\right)\right) .
\end{aligned}
$$

Therefore, $G_{1}$ satisfies 2 with an positive number $L=w^{2} e^{w / 2}, L_{1}=L_{2}=1 / 8$, and $c=d=1$.

Then, from Theorem 3, we see that if we choose positive weighted number $\beta$ satisfying $\beta>(1 / 8)+\sqrt{(1 / 32)+w^{2} e^{w / 2}}$, Hence, all the assumptions of Theorem 3 are satisfied; therefore, we have a unique intuitionistic fuzzy solution of problems (45)-(47).

We try to calculate the intuitionistic fuzzy solution of this problem by using the method of steps proposed in $[22,23]$.

We write the deterministic solution of the crisp equation as follows: $v=c e^{w(\lambda+\theta)}$.

Assume that the parametric form of corresponding intuitionistic fuzzy number $C$ is

$$
\begin{aligned}
& {[C]_{\alpha}=\left[C_{l}^{+}(\alpha), C_{r}^{+}(\alpha)\right],} \\
& {[C]^{\alpha}=\left[C_{l}^{-}(\alpha), C_{r}^{-}(\alpha)\right],}
\end{aligned}
$$

where it verifies the conditions of Lemma 1 .

The function $G_{1}:[0,(\pi / 2)] \times[0,1] \times \mathrm{IF}_{1} \longrightarrow \mathrm{IF}_{1}$ is defined as

$$
G_{1}\left(\lambda, \theta,\left\langle\phi_{1}, \phi_{2}\right\rangle_{(\lambda, \theta)}\right)=w^{2} e^{w / 2}\left\langle\phi_{1}, \phi_{2}\right\rangle\left(\lambda-\frac{1}{4}, \theta-\frac{1}{4}\right) .
$$

The $\alpha$-cuts of $G_{1}$ are

$$
\begin{aligned}
{\left[G_{1}\right]_{\alpha}=} & {\left[w^{2} e^{w / 2}\left[\left\langle\phi_{1}, \phi_{2}\right\rangle\left(\lambda-\frac{1}{4}, \theta-\frac{1}{4}\right)\right]_{l}^{+}(\alpha), w^{2} e^{w / 2}\right.} \\
& \left.\cdot\left[\left\langle\phi_{1}, \phi_{2}\right\rangle\left(\lambda-\frac{1}{4}, \theta-\frac{1}{4}\right)\right]_{r}^{+}(\alpha)\right] \\
{\left[G_{1}\right]^{\alpha}=} & {\left[w^{2} e^{w / 2}\left[\left\langle\phi_{1}, \phi_{2}\right\rangle\left(\lambda-\frac{1}{4}, \theta-\frac{1}{4}\right)\right]_{l}^{-}(\alpha), w^{2} e^{w / 2}\right.} \\
& \left.\cdot\left[\left\langle\phi_{1}, \phi_{2}\right\rangle\left(\lambda-\frac{1}{4}, \theta-\frac{1}{4}\right)\right]_{r}^{-}(\alpha)\right] .
\end{aligned}
$$

If we denote

$\left[\left\langle\phi_{1}, \phi_{2}\right\rangle(\lambda, \theta)\right]_{\alpha}=\left[\left[\left\langle\phi_{1}, \phi_{2}\right\rangle(\lambda, \theta)\right]_{l}^{+}(\alpha),\left[\left\langle\phi_{1}, \phi_{2}\right\rangle(\lambda, \theta)\right]_{r}^{+}(\alpha)\right]$, $\left[\left\langle\phi_{1}, \phi_{2}\right\rangle(\lambda, \theta)\right]^{\alpha}=\left[\left[\left\langle\phi_{1}, \phi_{2}\right\rangle(\lambda, \theta)\right]_{l}^{-}(\alpha),\left[\left\langle\phi_{1}, \phi_{2}\right\rangle(\lambda, \theta)\right]_{r}^{-}(\alpha)\right]$.

Then,

$$
\begin{aligned}
{\left[\frac{\partial^{2}\left\langle\phi_{1}, \phi_{2}\right\rangle(\lambda, \theta)}{\partial \lambda \partial \theta}\right]_{\alpha}=} & {\left[\left[\frac{\partial^{2}\left\langle\phi_{1}, \phi_{2}\right\rangle(\lambda, \theta)}{\partial \lambda \partial \theta}\right]_{l}^{+}(\alpha),\right.} \\
& \left.\cdot\left[\frac{\partial^{2}\left\langle\phi_{1}, \phi_{2}\right\rangle(\lambda, \theta)}{\partial \lambda \partial \theta}\right]_{r}^{+}(\alpha)\right], \\
{\left[\frac{\partial^{2}\left\langle\phi_{1}, \phi_{2}\right\rangle(\lambda, \theta)}{\partial \lambda \partial \theta}\right]^{\alpha}=} & {\left[\left[\frac{\partial^{2}\left\langle\phi_{1}, \phi_{2}\right\rangle(\lambda, \theta)}{\partial \lambda \partial \theta}\right]_{l}^{-}(\alpha),\right.} \\
& \left.\cdot\left[\frac{\partial^{2}\left\langle\phi_{1}, \phi_{2}\right\rangle(\lambda, \theta)}{\partial \lambda \partial \theta}\right]_{r}^{-}(\alpha)\right] .
\end{aligned}
$$

Therefore, we solve the following state-dependent delay partial functional differential equations for $(\lambda, \theta) \in[0,1] \times[0,1]:$

$$
\left\{\begin{array}{l}
{\left[\frac{\partial^{2}\left\langle\phi_{1}, \phi_{2}\right\rangle(\lambda, \theta)}{\partial \lambda \partial \theta}\right]_{l}^{+}(\alpha)=w^{2} e^{w / 2}\left[\left\langle\phi_{1}, \phi_{2}\right\rangle\left(\lambda-\frac{1}{4}, \theta-\frac{1}{4}\right)\right]_{l}^{+}(\alpha),} \\
{\left[\frac{\partial^{2}\left\langle\phi_{1}, \phi_{2}\right\rangle(\lambda, \theta)}{\partial \lambda \partial \theta}\right]_{r}^{+}(\alpha)=w^{2} e^{w / 2}\left[\left\langle\phi_{1}, \phi_{2}\right\rangle\left(\lambda-\frac{1}{4}, \theta-\frac{1}{4}\right)\right]_{r}^{+}(\alpha),} \\
{\left[\frac{\partial^{2}\left\langle\phi_{1}, \phi_{2}\right\rangle(\lambda, \theta)}{\partial \lambda \partial \theta}\right]_{l}^{-}(\alpha)=w^{2} e^{w / 2}\left[\left\langle\phi_{1}, \phi_{2}\right\rangle\left(\lambda-\frac{1}{4}, \theta-\frac{1}{4}\right)\right]_{l}^{-}(\alpha),} \\
{\left[\frac{\partial^{2}\left\langle\phi_{1}, \phi_{2}\right\rangle(\lambda, \theta)}{\partial \lambda \partial \theta}\right]_{r}^{-}(\alpha)=w^{2} e^{w / 2}\left[\left\langle\phi_{1}, \phi_{2}\right\rangle\left(\lambda-\frac{1}{4}, \theta-\frac{1}{4}\right)\right]_{r}^{-}(\alpha),}
\end{array}\right.
$$

with initial conditions for $\quad(\lambda, \theta t \in n[-(1 / 4)$, $1] q \times h[-(1 / 4), 1] \backslash x(0,1] 7 \times C(0,1]:$ 


$$
\left\{\begin{array}{c}
{\left[\left\langle\phi_{1}, \phi_{2}\right\rangle(\lambda, \theta)\right]_{l}^{+}(\alpha)=C_{l}^{+}\left(e^{w \lambda}+e^{w \theta}+\lambda \theta\right),} \\
{\left[\left\langle\phi_{1}, \phi_{2}\right\rangle(\lambda, \theta)\right]_{r}^{+}(\alpha)=C_{r}^{+}\left(e^{w \lambda}+e^{w \theta}+\lambda \theta\right),} \\
{\left[\left\langle\phi_{1}, \phi_{2}\right\rangle(\lambda, \theta)\right]_{l}^{-}(\alpha)=C_{l}^{-}\left(e^{w \lambda}+e^{w \theta}+\lambda \theta\right),} \\
{\left[\left\langle\phi_{1}, \phi_{2}\right\rangle(\lambda, \theta)\right]_{r}^{-}(\alpha)=C_{r}^{-}\left(e^{w \lambda}+e^{w \theta}+\lambda \theta\right),}
\end{array}\right.
$$

and for $(\lambda, \theta) \in[0,1] \times[0,1]$, we have

$$
\left\{\begin{array}{l}
{\left[\left\langle\phi_{1}, \phi_{2}\right\rangle(\lambda, 0)\right]_{l}^{+}(\alpha)+\frac{1}{8} \int_{0}^{\theta}\left[\left\langle\phi_{1}, \phi_{2}\right\rangle(\lambda, \sigma)\right]_{l}^{+}(\alpha) \mathrm{d} \sigma=C_{l}^{+}(\alpha) \frac{1}{8 w} e^{w \lambda}\left(e^{w \theta}+8 w-1\right),} \\
{\left[\left\langle\phi_{1}, \phi_{2}\right\rangle(\lambda, 0)\right]_{r}^{+}(\alpha)+\frac{1}{8} \int_{0}^{\theta}\left[\left\langle\phi_{1}, \phi_{2}\right\rangle(\lambda, \sigma)\right]_{r}^{+}(\alpha) \mathrm{d} \sigma=C_{r}^{+}(\alpha) \frac{1}{8 w} e^{w \lambda}\left(e^{w \theta}+8 w-1\right),} \\
{\left[\left\langle\phi_{1}, \phi_{2}\right\rangle(\lambda, 0)\right]_{l}^{-}(\alpha)+\frac{1}{8} \int_{0}^{\theta}\left[\left\langle\phi_{1}, \phi_{2}\right\rangle(\lambda, \sigma)\right]_{l}^{-}(\alpha) \mathrm{d} \sigma=C_{l}^{-}(\alpha) \frac{1}{8 w} e^{w \lambda}\left(e^{w \theta}+8 w-1\right),} \\
{\left[\left\langle\phi_{1}, \phi_{2}\right\rangle(\lambda, 0)\right]_{r}^{-}(\alpha)+\frac{1}{8} \int_{0}^{\theta}\left[\left\langle\phi_{1}, \phi_{2}\right\rangle(\lambda, \sigma)\right]_{r}^{-}(\alpha) \mathrm{d} \sigma=C_{r}^{-}(\alpha) \frac{1}{8 w} e^{w \lambda}\left(e^{w \theta}+8 w-1\right),} \\
{\left[\left\langle\phi_{1}, \phi_{2}\right\rangle(\lambda, 0)\right]_{l}^{+}(\alpha)+\frac{1}{8} \int_{0}^{\lambda}\left[\left\langle\phi_{1}, \phi_{2}\right\rangle(\zeta, \theta)\right]_{l}^{+}(\alpha) \mathrm{d} \zeta=C_{l}^{+}(\alpha) \frac{1}{8 w} e^{w \theta}\left(e^{w \lambda}+8 w-1\right),} \\
{\left[\left\langle\phi_{1}, \phi_{2}\right\rangle(\lambda, 0)\right]_{r}^{+}(\alpha)+\frac{1}{8} \int_{0}^{\lambda}\left[\left\langle\phi_{1}, \phi_{2}\right\rangle(\zeta, \theta)\right]_{r}^{+}(\alpha) \mathrm{d} \zeta=C_{r}^{+}(\alpha) \frac{1}{8 w} e^{w \theta}\left(e^{w \lambda}+8 w-1\right),} \\
{\left[\left\langle\phi_{1}, \phi_{2}\right\rangle(\lambda, 0)\right]_{l}^{-}(\alpha)+\frac{1}{8} \int_{0}^{\lambda}\left[\left\langle\phi_{1}, \phi_{2}\right\rangle(\zeta, \theta)\right]_{l}^{-}(\alpha) \mathrm{d} \zeta=C_{l}^{-}(\alpha) \frac{1}{8 w} e^{w \theta}\left(e^{w \lambda}+8 w-1\right),} \\
{\left[\left\langle\phi_{1}, \phi_{2}\right\rangle(\lambda, 0)\right]_{r}^{-}(\alpha)+\frac{1}{8} \int_{0}^{\lambda}\left[\left\langle\phi_{1}, \phi_{2}\right\rangle(\zeta, \theta)\right]_{r}^{-}(\alpha) \mathrm{d} \zeta=C_{r}^{-}(\alpha) \frac{1}{8 w} e^{w \theta}\left(e^{w \lambda}+8 w-1\right) .}
\end{array}\right.
$$

We get

Then,

$$
\begin{aligned}
{\left[\left\langle\phi_{1}, \phi_{2}\right\rangle(\lambda, \theta)\right]_{\alpha} } & =\left[C_{l}^{+}(\alpha) e^{w(\lambda+\theta)}, C_{r}^{+}(\alpha) e^{w(\lambda+\theta)}\right]\left[\left\langle\phi_{1}, \phi_{2}\right\rangle(\lambda, \theta)\right]^{\alpha} \\
& =\left[C_{l}^{-}(\alpha) e^{w(\lambda+\theta)}, C_{r}^{-}(\alpha) e^{w(\lambda+\theta)}\right] .
\end{aligned}
$$

Now we denote the families $N_{\alpha}$ and $N^{\alpha}$ as follows:

$$
\begin{cases}{\left[\left\langle\phi_{1}, \phi_{2}\right\rangle(\lambda, \theta)\right]_{l}^{+}(\alpha)=C_{l}^{+}(\alpha) e^{w(\lambda+\theta)},} & (\lambda, \theta) \in[0,1] \times[0,1], \\ {\left[\left\langle\phi_{1}, \phi_{2}\right\rangle(\lambda, \theta)\right]_{r}^{+}(\alpha)=C_{r}^{+}(\alpha) e^{w(\lambda+\theta)},} & (\lambda, \theta) \in[0,1] \times[0,1], \\ {\left[\left\langle\phi_{1}, \phi_{2}\right\rangle(\lambda, \theta)\right]_{l}^{-}(\alpha)=C_{l}^{-}(\alpha) e^{w(\lambda+\theta)},} & (\lambda, \theta) \in[0,1] \times[0,1], \\ {\left[\left\langle\phi_{1}, \phi_{2}\right\rangle(\lambda, \theta)\right]_{r}^{-}(\alpha)=C_{r}^{-}(\alpha) e^{w(\lambda+\theta)},} & (\lambda, \theta) \in[0,1] \times[0,1] .\end{cases}
$$

and the families $N_{\alpha}^{\prime}$ and $N^{\prime \alpha}$ by

$$
\begin{aligned}
& {\left[w^{2} e^{w / 2} C_{l}^{-}(\alpha) e^{w(\lambda+\theta)}, w^{2} e^{w / 2} C_{r}^{-}(\alpha) e^{w(\lambda+\theta)}\right]=N_{\alpha}^{\prime},} \\
& {\left[w^{2} e^{w / 2} C_{l}^{-}(\alpha) e^{w(\lambda+\theta)}, w^{2} e^{w / 2} C_{r}^{-}(\alpha) e^{w(\lambda+\theta)}\right]=N^{\prime \alpha}}
\end{aligned}
$$


It is easy to see that $\left(N_{\alpha}, N^{\alpha}\right)$ and $\left(N_{\alpha}^{\prime}, N^{\prime \alpha}\right)$ satisfy the conditions of Proposition 1, and by applying Lemma 1, we can build the intuitionistic fuzzy solution $\left\langle\phi_{1}, \phi_{2}\right\rangle$ $(\lambda, \theta) \in \mathrm{IF}_{1}$ for equations (52)-(54) for every $\alpha \in[0,1]$ as follows:

$$
\begin{aligned}
& {\left[\left\langle\phi_{1}, \phi_{2}\right\rangle(\lambda, \theta)\right]_{\alpha}=\left[C_{l}^{+}(\alpha) e^{w(\lambda+\theta)}, C_{r}^{+}(\alpha) e^{w(\lambda+\theta)}\right],} \\
& {\left[\left\langle\phi_{1}, \phi_{2}\right\rangle(\lambda, \theta)\right]^{\alpha}=\left[C_{l}^{-}(\alpha) e^{w(\lambda+\theta)}, C_{r}^{-}(\alpha) e^{w(\lambda+\theta)}\right] .}
\end{aligned}
$$

Then, $\left\langle\phi_{1}, \phi_{2}\right\rangle(x, y)$ is an intuitionistic fuzzy solution which satisfies the initial conditions (53)-(54) and is written as follows:

$$
\left\langle\phi_{1}, \phi_{2}\right\rangle(x, y)=C e^{w(\lambda+\theta)} .
$$

Figures 1 and 2 show the membership and nonmembership functions of triangular intuitionistic fuzzy number $C=(-0.75,0,0.75 ;-2,0,2)$ and the simulation of $\alpha$-cuts of the intuitionistic fuzzy solution $\left\langle\phi_{1}, \phi_{2}\right\rangle(x, y)$ at some values of $(\lambda, \theta)$ with $w=0.45$.

In Figure 3 we present the surface of the intuitionistic fuzzy solution with triangular intuitionistic fuzzy numbers $C=(-0.75,0,0.75 ;-2,0,2)$ and $w=0.45$.

Example 2. Now, we present a second example to illustrate Theorem 4. Consider the following state-dependent delay intuitionistic fuzzy partial functional differential equations in the general form:

$$
\begin{aligned}
\frac{\partial^{2}\left\langle\phi_{1}, \phi_{2}\right\rangle(\lambda, \theta)}{\partial \lambda \partial \theta}= & \frac{\partial\left((1 / 2) e^{-(2 / 3)}\left\langle\phi_{1}, \phi_{2}\right\rangle(\lambda, \theta)\right)}{\partial \theta}-\frac{1}{2}\left\langle\phi_{1}, \phi_{2}\right\rangle \\
& \cdot\left(\lambda-\frac{1}{3}, \theta-\frac{1}{3}\right)+c_{1} e^{\lambda+\theta}+\frac{1}{2} c_{2} \cos \left(\lambda-\frac{1}{3}\right) .
\end{aligned}
$$

And, the integral boundary initial conditions are

$$
\begin{aligned}
& \left\langle\phi_{1}, \phi_{2}\right\rangle(\lambda, 0)+\frac{1}{2} \int_{0}^{\theta}\left\langle\phi_{1}, \phi_{2}\right\rangle(\lambda, \sigma) \mathrm{d} \sigma=\frac{c_{1}}{2} e^{\lambda}\left(1+e^{\theta}\right)+c_{2} \cos (\lambda)\left(1+\frac{\theta}{2}\right), \\
& \left\langle\phi_{1}, \phi_{2}\right\rangle(0, \theta)+\frac{1}{2} \int_{0}^{\lambda}\left\langle\phi_{1}, \phi_{2}\right\rangle(\zeta, \theta) \mathrm{d} \zeta=\frac{c_{1}}{2} e^{\theta}\left(1+e^{\lambda}\right)+c_{2}\left(1+\frac{\sin (\lambda)}{2}\right),
\end{aligned}
$$

where $c_{1}$ and $c_{2} \in \mathrm{IF}_{1}$ are triangular intuitionistic fuzzy numbers and $(\lambda, \theta) \in[0,(\pi / 2)] \times[0,1]$, and for $\left(\lambda, \theta t \in n q-1 / 3, \pi / 2 h_{\times} x 7-1 / 3,1 C ; \backslash 0, \pi / 2 \times 0,1\right]$, the initial condition is

$$
\left\langle\phi_{1}, \phi_{2}\right\rangle(\lambda, \theta)=c_{1}\left(e^{\lambda}+\lambda \theta\right)+c_{2}\left(e^{\theta}+\cos (\lambda \theta)\right) .
$$

Since

$$
\begin{aligned}
& G_{2}\left(\lambda, \theta,\left\langle\phi_{1}, \phi_{2}\right\rangle_{(\lambda, \theta)}\right)=-\frac{1}{2}\left\langle\phi_{1}, \phi_{2}\right\rangle\left(\lambda-\frac{1}{3}, \theta-\frac{1}{3}\right) \\
& +c_{1} e^{\lambda+\theta}+\frac{1}{2} c_{2} \cos \left(\lambda-\frac{1}{3}\right),
\end{aligned}
$$

it verifies conditions 1 and 2 of Theorem 1 .

Indeed, it is easy to see that $G_{2}$ is continuous:

$$
\begin{aligned}
& d_{\infty}\left(G_{2}\left(\lambda, \theta,\left\langle\phi_{1}, \phi_{2}\right\rangle_{(\lambda, \theta)}\right), G_{2}\left(\lambda, \theta,\left\langle\phi_{1}^{\prime}, \phi_{2}^{\prime}\right\rangle_{(\lambda, \theta)}\right)\right) \\
& \quad \leq \frac{1}{2} d_{\infty}\left(\left\langle\phi_{1}, \phi_{2}\right\rangle_{(\lambda, \theta)}\left(-\frac{1}{3},-\frac{1}{3}\right),\left\langle\phi_{1}^{\prime}, \phi_{2}^{\prime}\right\rangle_{(\lambda, \theta)}\left(-\frac{1}{3},-\frac{1}{3}\right)\right),
\end{aligned}
$$

so the assumptions are satisfied with a positive number $L=1 / 2, \quad L_{1}=L_{2}=1 / 2, \quad c=\pi / 2, \quad d=1, \quad$ and $\quad q(\lambda, \theta)=$ $(1 / 2) e^{-(2 / 3)}$.

If we choose the positive weighted number $\beta$ satisfying $\beta^{2}-\beta\left(1+e^{-(2 / 3)}\right)-(3 / 4)>0$, then $\beta>(1 / 2)+\left(e^{-(2 / 3)} / 2\right)+$ $\sqrt{1+(1 / 4) e^{-(4 / 3)}+(1 / 2) e^{-(2 / 3)}}$; hence, all the hypothesis of
Theorem 4 hold; therefore, we have a unique intuitionistic fuzzy solution of equations (72)-(74).

We will use the method of steps proposed in $[22,23]$ to find an intuitionistic fuzzy solution of equations (72)-(74).

The classical solution of the crisp equation is $w=C_{1} e^{\lambda+\theta}+C_{2} \cos (\lambda)$.

Assume that the parametric forms of corresponding intuitionistic fuzzy number $c_{i}(i=1,2)$ are

$$
\begin{aligned}
& {\left[c_{i}\right]_{\alpha}=\left[c_{i l}^{+}(\alpha), c_{i r}^{+}(\alpha)\right],} \\
& {\left[c_{i}\right]^{\alpha}=\left[c_{i l}^{-}(\alpha), c_{i r}^{-}(\alpha)\right],}
\end{aligned}
$$

where they verify the conditions of Lemma 1 .

The function $G_{2}:[0,(\pi / 2)] \times[0,1] \times \mathrm{IF}_{1} \longrightarrow \mathrm{IF}_{1}$ is defined by

$$
\begin{aligned}
& G_{2}\left(\lambda, \theta,\left\langle\phi_{1}, \phi_{2}\right\rangle_{(\lambda, \theta)}\right)=-\frac{1}{2}\left\langle\phi_{1}, \phi_{2}\right\rangle\left(\lambda-\frac{1}{3}, \theta-\frac{1}{3}\right)+c_{1} e^{\lambda+\theta} \\
& +\frac{1}{2} c_{2} \cos \left(\lambda-\frac{1}{3}\right) \text {. }
\end{aligned}
$$

The $\alpha$-cuts of $G_{2}$ are

$$
\begin{aligned}
{\left[G_{2}\right]_{\alpha} } & =\left[G_{2 l}^{+}(\alpha), G_{2 r}^{+}(\alpha)\right], \\
{\left[G_{2}\right]^{\alpha} } & =\left[G_{2 l}^{-}(\alpha), G_{2 r}^{-}(\alpha)\right],
\end{aligned}
$$

where 

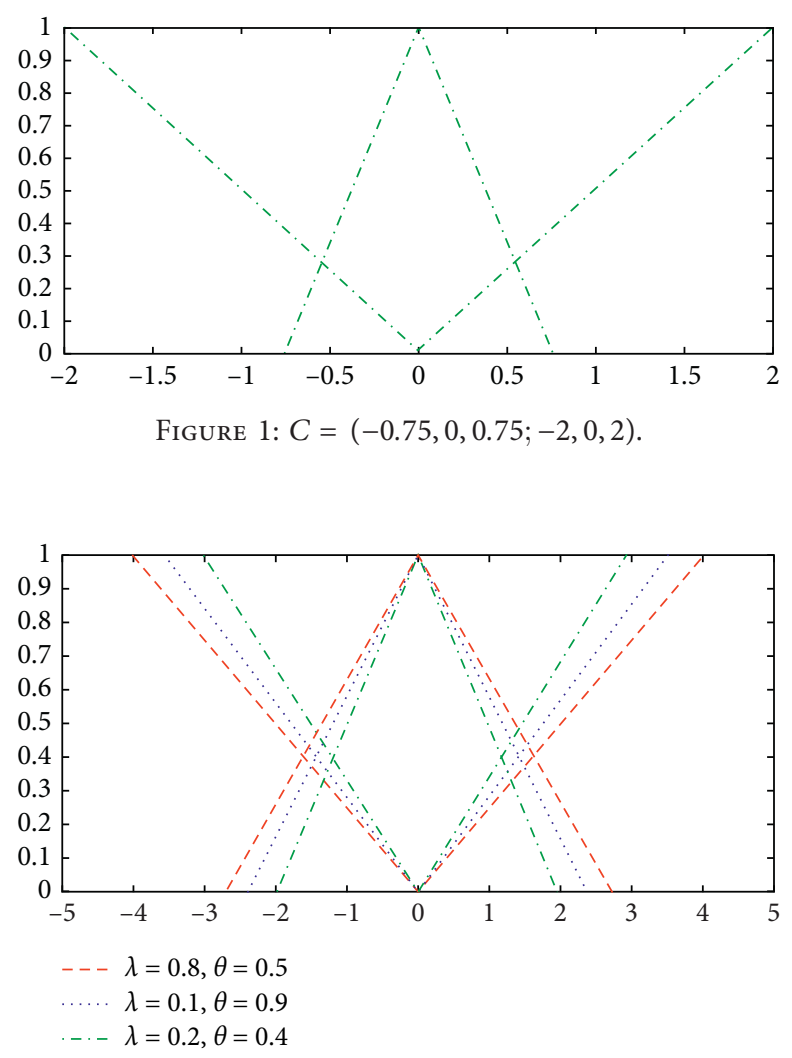

Figure 2: The curve of intuitionistic fuzzy solutions $\left\langle\phi_{1}, \phi_{2}\right\rangle(x, y)$ at different values of $(\lambda, \theta)$.

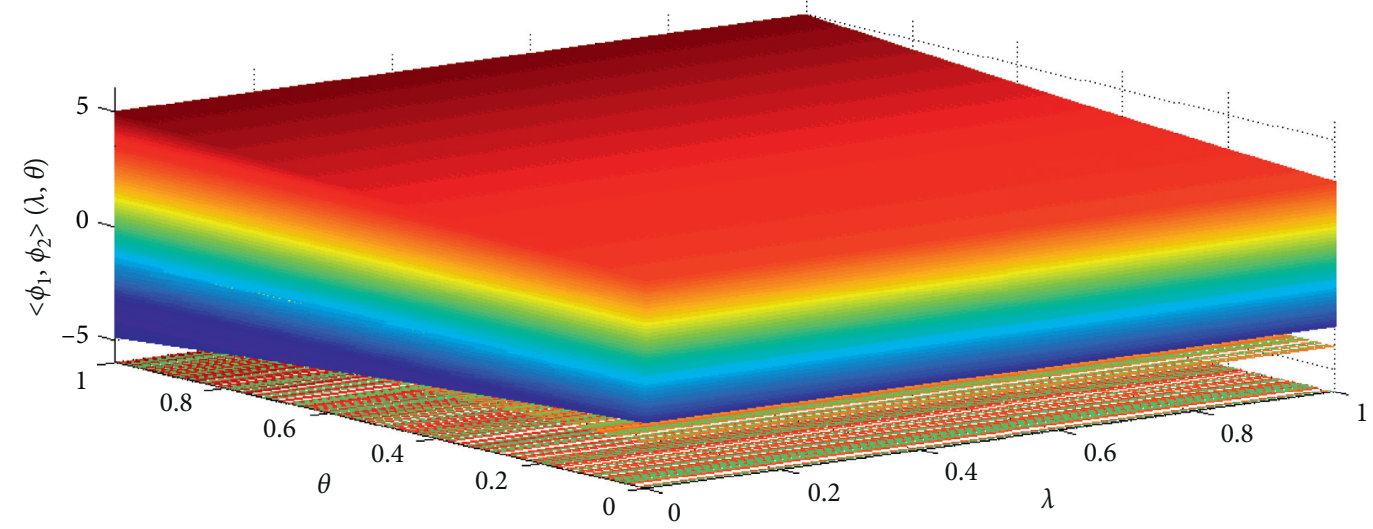

FIGURE 3: The surface of the intuitionistic fuzzy solution.

$$
\begin{aligned}
& G_{2 l}^{+}(\alpha)=-\frac{1}{2}\left[\left\langle\phi_{1}, \phi_{2}\right\rangle\left(\lambda-\frac{1}{3}, \theta-\frac{1}{3}\right)\right]_{l}^{+}(\alpha)+c_{1 l}^{+}(\alpha) e^{\lambda+\theta}+\frac{1}{2} c_{2 l}^{+}(\alpha) \cos \left(\lambda-\frac{1}{3}\right), \\
& G_{2 r}^{+}(\alpha)=-\frac{1}{2}\left[\left\langle\phi_{1}, \phi_{2}\right\rangle\left(\lambda-\frac{1}{3}, \theta-\frac{1}{3}\right)\right]_{r}^{+}(\alpha)+c_{1 r}^{+}(\alpha) e^{\lambda+\theta}+\frac{1}{2} c_{2 r}^{+}(\alpha) \cos \left(\lambda-\frac{1}{3}\right), \\
& G_{2 l}^{-}(\alpha)=-\frac{1}{2}\left[\left\langle\phi_{1}, \phi_{2}\right\rangle\left(\lambda-\frac{1}{3}, \theta-\frac{1}{3}\right)\right]_{l}^{-}(\alpha)+c_{1 l}^{-}(\alpha) e^{\lambda+\theta}+\frac{1}{2} c_{2 l}^{-}(\alpha) \cos \left(\lambda-\frac{1}{3}\right), \\
& G_{2 r}^{-}(\alpha)=-\frac{1}{2}\left[\left\langle\phi_{1}, \phi_{2}\right\rangle\left(\lambda-\frac{1}{3}, \theta-\frac{1}{3}\right)\right]_{r}^{-}(\alpha)+c_{1 r}^{-}(\alpha) e^{\lambda+\theta}+\frac{1}{2} c_{2 r}^{-}(\alpha) \cos \left(\lambda-\frac{1}{3}\right) .
\end{aligned}
$$




\section{If}

$\left[\left\langle\phi_{1}, \phi_{2}\right\rangle(\lambda, \theta)\right]_{\alpha}=\left[\left[\left\langle\phi_{1}, \phi_{2}\right\rangle(\lambda, \theta)\right]_{l}^{+}(\alpha),\left[\left\langle\phi_{1}, \phi_{2}\right\rangle(\lambda, \theta)\right]_{r}^{+}(\alpha)\right]$ $\left[\left\langle\phi_{1}, \phi_{2}\right\rangle(\lambda, \theta)\right]^{\alpha}=\left[\left[\left\langle\phi_{1}, \phi_{2}\right\rangle(\lambda, \theta)\right]_{l}^{-}(\alpha),\left[\left\langle\phi_{1}, \phi_{2}\right\rangle(\lambda, \theta)\right]_{r}^{-}(\alpha)\right]$,

then

$$
\begin{aligned}
{\left[\frac{\partial^{2}\left\langle\phi_{1}, \phi_{2}\right\rangle(\lambda, \theta)}{\partial \lambda \partial \theta}\right]_{\alpha}=} & {\left[\left[\frac{\partial^{2}\left\langle\phi_{1}, \phi_{2}\right\rangle(\lambda, \theta)}{\partial \lambda \partial \theta}\right]_{l}^{+}(\alpha)\right.} \\
& \left.\cdot\left[\frac{\partial^{2}\left\langle\phi_{1}, \phi_{2}\right\rangle(\lambda, \theta)}{\partial \lambda \partial \theta}\right]_{r}^{+}(\alpha)\right], \\
{\left[\frac{\partial^{2}\left\langle\phi_{1}, \phi_{2}\right\rangle(\lambda, \theta)}{\partial \lambda \partial \theta}\right]^{\alpha}=} & {\left[\left[\frac{\partial^{2}\left\langle\phi_{1}, \phi_{2}\right\rangle(\lambda, \theta)}{\partial \lambda \partial \theta}\right]_{l}^{-}(\alpha)\right.} \\
& \left.\cdot\left[\frac{\partial^{2}\left\langle\phi_{1}, \phi_{2}\right\rangle(\lambda, \theta)}{\partial \lambda \partial \theta}\right]_{r}^{-}(\alpha)\right] .
\end{aligned}
$$

Then, we solve the following state-dependent delay partial functional differential equations:

$$
\left\{\begin{array}{l}
{\left[\frac{\partial^{2}\left\langle\phi_{1}, \phi_{2}\right\rangle(\lambda, \theta)}{\partial \lambda \partial \theta}\right]_{l}^{+}(\alpha)=\left[\frac{\partial\left((1 / 2) e^{-(2 / 3)}\left\langle\phi_{1}, \phi_{2}\right\rangle(\lambda, \theta)\right)}{\partial \theta}\right]_{l}^{+}(\alpha)-\frac{1}{2}\left[\left\langle\phi_{1}, \phi_{2}\right\rangle\left(\lambda-\frac{1}{3}, \theta-\frac{1}{3}\right)\right]_{l}^{+}(\alpha)+c_{1 l}^{+}(\alpha) e^{\lambda+\theta}+\frac{1}{2} c_{2 l}^{+}(\alpha) \cos \left(\lambda-\frac{1}{3}\right),} \\
{\left[\frac{\partial^{2}\left\langle\phi_{1}, \phi_{2}\right\rangle(\lambda, \theta)}{\partial \lambda \partial \theta}\right]_{r}^{+}(\alpha)=\left[\frac{\partial\left((1 / 2) e^{-(2 / 3)}\left\langle\phi_{1}, \phi_{2}\right\rangle(\lambda, \theta)\right)}{\partial \theta}\right]_{r}^{+}(\alpha)-\frac{1}{2}\left[\left\langle\phi_{1}, \phi_{2}\right\rangle\left(\lambda-\frac{1}{3}, \theta-\frac{1}{3}\right)\right]_{r}^{+}(\alpha)+c_{1 r}^{+}(\alpha) e^{\lambda+\theta}+\frac{1}{2} c_{2 r}^{+}(\alpha) \cos \left(\lambda-\frac{1}{3}\right),} \\
{\left[\frac{\partial^{2}\left\langle\phi_{1}, \phi_{2}\right\rangle(\lambda, \theta)}{\partial \lambda \partial \theta}\right]_{l}^{-}(\alpha)=\left[\frac{\partial\left((1 / 2) e^{-(3 / 4)}\left\langle\phi_{1}, \phi_{2}\right\rangle(\lambda, \theta)\right)}{\partial \theta}\right]_{l}^{-}(\alpha)-\frac{1}{2}\left[\left\langle\phi_{1}, \phi_{2}\right\rangle\left(\lambda-\frac{1}{3}, \theta-\frac{1}{3}\right)\right]_{l}^{-}(\alpha)+c_{1 l}^{-}(\alpha) e^{\lambda+\theta}+\frac{1}{2} c_{2 l}^{-}(\alpha) \cos \left(\lambda-\frac{1}{3}\right),} \\
{\left[\frac{\partial^{2}\left\langle\phi_{1}, \phi_{2}\right\rangle(\lambda, \theta)}{\partial \lambda \partial \theta}\right]_{r}^{-}(\alpha)=\left[\frac{\partial\left((1 / 2) e^{-(2 / 3)}\left\langle\phi_{1}, \phi_{2}\right\rangle(\lambda, \theta)\right)}{\partial \theta}\right]_{r}^{-}(\alpha)-\frac{1}{2}\left[\left\langle\phi_{1}, \phi_{2}\right\rangle\left(\lambda-\frac{1}{3}, \theta-\frac{1}{3}\right)\right]_{r}^{-}(\alpha)+c_{1 r}^{-}(\alpha) e^{\lambda+\theta}+\frac{1}{2} c_{2 r}^{-}(\alpha) \cos \left(\lambda-\frac{1}{3}\right),}
\end{array}\right.
$$

where $(\lambda, \theta) \in[0,(\pi / 2)] \times[0,1]$ with initial conditions for $\left(\lambda, \theta t \in n q-1 / 2, \pi / 2 h_{\times} x 7-1 / 2,1 C ; \backslash 0, \pi / 2 \times 0,1\right]$ :

$$
\left\{\begin{array}{l}
{\left[\left\langle\phi_{1}, \phi_{2}\right\rangle(\lambda, \theta)\right]_{l}^{+}(\alpha)=c_{1 l}^{+}(\alpha)\left(e^{\lambda}+\lambda \theta\right)+c_{2 l}^{+}(\alpha)\left(e^{\theta}+\cos (\lambda \theta)\right),} \\
{\left[\left\langle\phi_{1}, \phi_{2}\right\rangle(\lambda, \theta)\right]_{r}^{+}(\alpha)=c_{1 r}^{+}(\alpha)\left(e^{\lambda}+\lambda \theta\right)+c_{2 r}^{+}(\alpha)\left(e^{\theta}+\cos (\lambda \theta)\right),} \\
{\left[\left\langle\phi_{1}, \phi_{2}\right\rangle(\lambda, \theta)\right]_{l}^{-}(\alpha)=c_{1 l}^{-}(\alpha)\left(e^{\lambda}+\lambda \theta\right)+c_{2 l}^{-}(\alpha)\left(e^{\theta}+\cos (\lambda \theta)\right),} \\
{\left[\left\langle\phi_{1}, \phi_{2}\right\rangle(\lambda, \theta)\right]_{r}^{-}(\alpha)=c_{1 r}^{-}(\alpha)\left(e^{\lambda}+\lambda \theta\right)+c_{2 r}^{-}(\alpha)\left(e^{\theta}+\cos (\lambda \theta)\right),}
\end{array}\right.
$$

and for $(\lambda, \theta) \in[0,(\pi / 2)] \times[0,1]$, 


$$
\left\{\begin{array}{l}
{\left[\left\langle\phi_{1}, \phi_{2}\right\rangle(\lambda, 0)\right]_{l}^{+}(\alpha)+\frac{1}{2} \int_{0}^{\theta}\left[\left\langle\phi_{1}, \phi_{2}\right\rangle(\lambda, \sigma)\right]_{l}^{+}(\alpha) \mathrm{d} \sigma=\frac{1}{2} c_{1 l}^{+}(\alpha) e^{\lambda}\left(1+e^{\theta}\right)+c_{2 l}^{+}(\alpha) \cos (\lambda)\left(1+\frac{\theta}{2}\right),} \\
{\left[\left\langle\phi_{1}, \phi_{2}\right\rangle(\lambda, 0)\right]_{r}^{+}(\alpha)+\frac{1}{2} \int_{0}^{\theta}\left[\left\langle\phi_{1}, \phi_{2}\right\rangle(\lambda, \sigma)\right]_{r}^{+}(\alpha) \mathrm{d} \sigma=\frac{1}{2} c_{1 r}^{+}(\alpha) e^{\lambda}\left(1+e^{\theta}\right)+c_{2 r}^{+}(\alpha) \cos (\lambda)\left(1+\frac{\theta}{2}\right)} \\
{\left[\left\langle\phi_{1}, \phi_{2}\right\rangle(\lambda, 0)\right]_{l}^{-}(\alpha)+\frac{1}{2} \int_{0}^{\theta}\left[\left\langle\phi_{1}, \phi_{2}\right\rangle(\lambda, \sigma)\right]_{l}^{-}(\alpha) \mathrm{d} \sigma=\frac{1}{2} c_{1 l}^{-}(\alpha) e^{\lambda}\left(1+e^{\theta}\right)+c_{2 l}^{-}(\alpha) \cos (\lambda)\left(1+\frac{\theta}{2}\right),} \\
{\left[\left\langle\phi_{1}, \phi_{2}\right\rangle(\lambda, 0)\right]_{r}^{-}(\alpha)+\frac{1}{2} \int_{0}^{\theta}\left[\left\langle\phi_{1}, \phi_{2}\right\rangle(\lambda, \sigma)\right]_{r}^{-}(\alpha) \mathrm{d} \sigma=\frac{1}{2} c_{1 r}^{-}(\alpha) e^{\lambda}\left(1+e^{\theta}\right)+c_{2 r}^{-}(\alpha) \cos (\lambda)\left(1+\frac{\theta}{2}\right)} \\
{\left[\left\langle\phi_{1}, \phi_{2}\right\rangle(0, \theta)\right]_{l}^{+}(\alpha)+\frac{1}{2} \int_{0}^{\lambda}\left[\left\langle\phi_{1}, \phi_{2}\right\rangle(\zeta, \theta)\right]_{l}^{+}(\alpha) \mathrm{d} \zeta=\frac{1}{2} c_{1 l}^{+}(\alpha) e^{\theta}\left(1+e^{\lambda}\right)+c_{2 l}^{+}(\alpha)\left(1+\frac{\sin (\lambda)}{2}\right),} \\
{\left[\left\langle\phi_{1}, \phi_{2}\right\rangle(0, \theta)\right]_{r}^{+}(\alpha)+\frac{1}{2} \int_{0}^{\lambda}\left[\left\langle\phi_{1}, \phi_{2}\right\rangle(\zeta, \theta)\right]_{r}^{+}(\alpha) \mathrm{d} \zeta=\frac{1}{2} c_{1 r}^{+}(\alpha) e^{\theta}\left(1+e^{\lambda}\right)+c_{2 r}^{+}(\alpha)\left(1+\frac{\sin (\lambda)}{2}\right),} \\
{\left[\left\langle\phi_{1}, \phi_{2}\right\rangle(0, \theta)\right]_{l}^{-}(\alpha)+\frac{1}{2} \int_{0}^{\lambda}\left[\left\langle\phi_{1}, \phi_{2}\right\rangle(\zeta, \theta)\right]_{l}^{-}(\alpha) \mathrm{d} \zeta=\frac{1}{2} c_{1 l}^{-}(\alpha) e^{\theta}\left(1+e^{\lambda}\right)+c_{2 l}^{-}(\alpha)\left(1+\frac{\sin (\lambda)}{2}\right),} \\
{\left[\left\langle\phi_{1}, \phi_{2}\right\rangle(0, \theta)\right]_{r}^{-}(\alpha)+\frac{1}{2} \int_{0}^{\lambda}\left[\left\langle\phi_{1}, \phi_{2}\right\rangle(\zeta, \theta)\right]_{r}^{-}(\alpha) \mathrm{d} \zeta=\frac{1}{2} c_{1 r}^{-}(\alpha) e^{\theta}\left(1+e^{\lambda}\right)+c_{2 r}^{-}(\alpha)\left(1+\frac{\sin (\lambda)}{2}\right)}
\end{array}\right.
$$

We get

$$
\begin{cases}{\left[\left\langle\phi_{1}, \phi_{2}\right\rangle(\lambda, \theta)\right]_{l}^{+}(\alpha)=c_{1 l}^{+}(\alpha) e^{\lambda+\theta}+c_{2 l}^{+}(\alpha) \cos (\lambda),} & (\lambda, \theta) \in\left[0, \frac{\pi}{2}\right] \times[0,1], \\ {\left[\left\langle\phi_{1}, \phi_{2}\right\rangle(\lambda, \theta)\right]_{r}^{+}(\alpha)=c_{1 r}^{+}(\alpha) e^{\lambda+\theta}+c_{2 r}^{+}(\alpha) \cos (\lambda),} & (\lambda, \theta) \in\left[0, \frac{\pi}{2}\right] \times[0,1], \\ {\left[\left\langle\phi_{1}, \phi_{2}\right\rangle(\lambda, \theta)\right]_{l}^{-}(\alpha)=c_{1 l}^{-}(\alpha) e^{\lambda+\theta}+c_{2 l}^{-}(\alpha) \cos (\lambda),} & (\lambda, \theta) \in\left[0, \frac{\pi}{2}\right] \times[0,1], \\ {\left[\left\langle\phi_{1}, \phi_{2}\right\rangle(\lambda, \theta)\right]_{r}^{-}(\alpha)=c_{1 r}^{-}(\alpha) e^{\lambda+\theta}+c_{2 r}^{-}(\alpha) \cos (\lambda),} & (\lambda, \theta) \in\left[0, \frac{\pi}{2}\right] \times[0,1] .\end{cases}
$$

Then,

$$
\begin{aligned}
& {\left[\left\langle\phi_{1}, \phi_{2}\right\rangle(\lambda, \theta)\right]_{\alpha}=\left[c_{1 l}^{+}(\alpha) e^{\lambda+\theta}+c_{2 l}^{+}(\alpha) \cos (\lambda), c_{1 r}^{+}(\alpha) e^{\lambda+\theta}+c_{2 r}^{+}(\alpha) \cos (\lambda)\right],} \\
& {\left[\left\langle\phi_{1}, \phi_{2}\right\rangle(\lambda, \theta)\right]^{\alpha}=\left[c_{1 l}^{-}(\alpha) e^{\lambda+\theta}+c_{2 l}^{-}(\alpha) \cos (\lambda), c_{1 r}^{-}(\alpha) e^{\lambda+\theta}+c_{2 r}^{-}(\alpha) \cos (\lambda)\right] .}
\end{aligned}
$$

Now we denote 


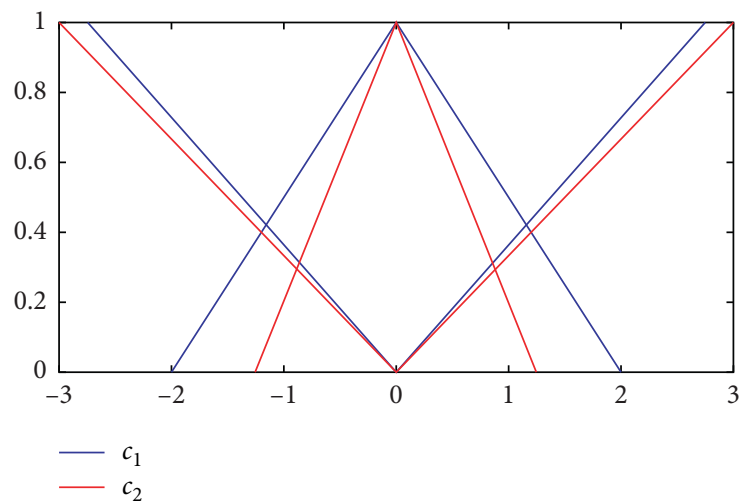

Figure $4: c_{1}=(-2,0,2 ;-2.75,0,2.75)$ and $c_{2}=(-1.25,0,1.25 ;-3,0,3)$.

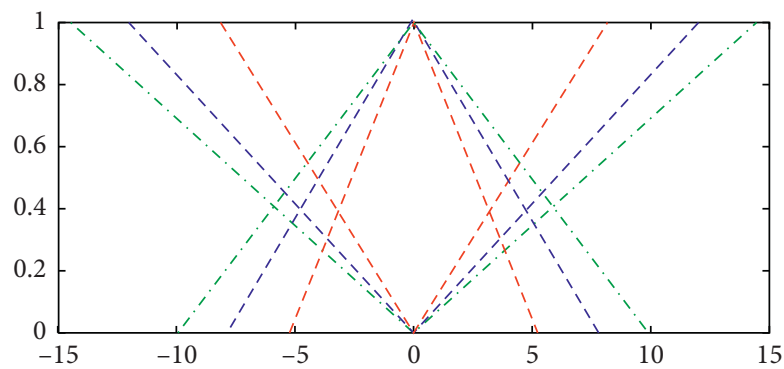

$--\lambda=0.5, \theta=0.2$

$--\lambda=0.3, \theta=0.9$

-. $\lambda=0.8, \theta=0.7$

Figure 5: $c_{1}=(-2,0,2 ;-2.75,0,2.75)$ and $c_{2}=(-1.25,0,1.25 ;-3,0,3)$.

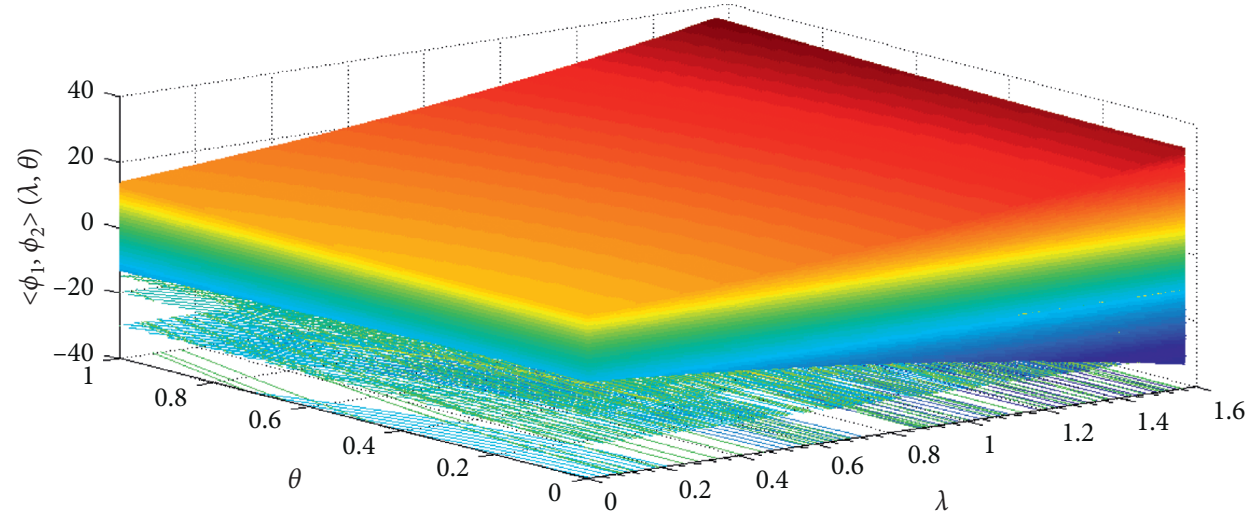

FIGURE 6: The surface of the intuitionistic fuzzy solution.

$\left[c_{1 l}^{+}(\alpha) e^{\lambda+\theta}+c_{2 l}^{+}(\alpha) \cos (\lambda), c_{1 r}^{+}(\alpha) e^{\lambda+\theta}+c_{2 r}^{+}(\alpha) \cos (\lambda)\right]=N_{\alpha}$, $\left[c_{1 l}^{-}(\alpha) e^{\lambda+\theta}+c_{2 l}^{-}(\alpha) \cos (\lambda), c_{1 r}^{-}(\alpha) e^{\lambda+\theta}+c_{2 r}^{-}(\alpha) \cos (\lambda)\right]=N^{\alpha}$,

$$
\begin{aligned}
& {\left[c_{1 l}^{+}(\alpha) e^{\lambda+\theta}, c_{1 r}^{+}(\alpha) e^{\lambda+\theta}\right]=N_{\alpha}^{\prime},} \\
& {\left[c_{1 l}^{-}(\alpha) e^{\lambda+\theta}, c_{1 r}^{-}(\alpha) e^{\lambda+\theta}\right]=N^{\prime \alpha} .}
\end{aligned}
$$

It easy to see that $\left(N_{\alpha}, N^{\alpha}\right)$ and $\left(N_{\alpha}^{\prime}, N^{\prime \alpha}\right)$ satisfy the conditions of Proposition 1, and by Lemma 1, we can build the intuitionistic fuzzy solution $\left\langle\phi_{1}, \phi_{2}\right\rangle(\lambda, \theta) \in \mathrm{IF}_{1}$ for equations (52)-(54) by the form as follows for every $\alpha \in[0,1]$ :

$$
\begin{aligned}
{\left[\left\langle\phi_{1}, \phi_{2}\right\rangle(\lambda, \theta)\right]_{\alpha}=} & {\left[c_{1 l}^{+}(\alpha) e^{\lambda+\theta}+c_{2 l}^{+}(\alpha) \cos (\lambda), c_{1 r}^{+}(\alpha) e^{\lambda+\theta}\right.} \\
& \left.+c_{2 r}^{+}(\alpha) \cos (\lambda)\right] \\
{\left[\left\langle\phi_{1}, \phi_{2}\right\rangle(\lambda, \theta)\right]^{\alpha}=} & {\left[c_{1 l}^{-}(\alpha) e^{\lambda+\theta}+c_{2 l}^{-}(\alpha) \cos (\lambda), c_{1 r}^{-}(\alpha) e^{\lambda+\theta}\right.} \\
& \left.+c_{2 r}^{-}(\alpha) \cos (\lambda)\right]
\end{aligned}
$$


Then, $\left\langle\phi_{1}, \phi_{2}\right\rangle(x, y)$ is an intuitionistic fuzzy solution which verifies the initial conditions (53)-(54) and can be written as follows:

$$
\left\langle\phi_{1}, \phi_{2}\right\rangle(x, y)=c_{1} e^{\lambda+\theta}+c_{2} \cos (\lambda) .
$$

Figures 4 and 5 represent the membership and nonmembership functions of triangular intuitionistic fuzzy numbers $\quad c_{1}=(-2,0,2 ;-2.75,0,2.75)$ and $c_{2}=(-1.25$, $0,1.25 ;-3,0,3)$ and the simulation of $\alpha$-cuts of the intuitionistic fuzzy solution $\left\langle\phi_{1}, \phi_{2}\right\rangle(x, y)$ at some values of $(\lambda, \theta)$.

In Figure 6, we show the surface of the intuitionistic fuzzy solution with triangular intuitionistic fuzzy numbers $c_{1}=(-2,0,2 ;-2.75,0,2.75) \quad$ and $\quad c_{2}=(-1.25,0,1.25$; $-3,0,3)$.

\section{Conclusion}

This research work proposed an integral boundary value problem for an intuitionistic fuzzy partial functional differential equation with state-dependent delay. We have proved the existence-uniqueness of intuitionistic fuzzy solutions for these problems with integral boundary conditions by applying the Banach fixed point theorem. These results are illustrated by numerical examples. Studying local and nonlocal intuitionistic fuzzy delay partial differential equations is the next step that will be considered.

\section{Data Availability}

No data were used to support this study.

\section{Conflicts of Interest}

The authors declare that they have no conflicts of interest.

\section{Authors' Contributions}

All authors are responsible for study conception and design, data collection, analysis and interpretation of results, and manuscript preparation.

\section{References}

[1] L. A. Zadeh, "Fuzzy sets," Information and Control, vol. 8, no. 3, pp. 338-353, 1965.

[2] K. T. Atanassov, Intuitionistic Fuzzy Sets, Academic Press, New York, NY, USA, 1983.

[3] K. T. Atanassov, "Intuitionistic fuzzy sets," Fuzzy Sets and Systems, vol. 20, pp. 87-96, 1986.

[4] P. Melo-Pinto, T. Kim, K. Atanassov, E. Sotirova, A. Shannon, and M. Krawczak, "Generalized net model of e-learning evaluation with intuitionistic fuzzy estimations," in Issues in the Representation and Processing of Uncertain and Imprecise Informationpp. 241-249, Warszawa, Poland, 2005.

[5] S. Sotirov, E. Sotirova, P. Melin, O. Castillo, and K. Atanassov, "Modular neural network preprocessing procedure with intuitionistic fuzzy intercriteria analysis method," in Flexible Query Answering Systems 2015, T. Andreasen, Ed., pp. 175186, Springer, Cham, Switzerland, 2016.
[6] S. K. De, R. Biswas, and A. R. Roy, "An application of intuitionistic fuzzy sets in medical diagnosis," Fuzzy Sets Systems, vol. 117, pp. 209-213, 2001.

[7] A. Kharal, "Homeopathic drug selection using intuitionistic fuzzy sets," Homeopathy, vol. 98, pp. 35-39, 2009.

[8] D. F. Li and C. T. Cheng, "New similarity measures of intuitionistic fuzzy sets and application to pattern recognitions," Pattern Recognition Letters, vol. 23, pp. 221-225, 2002.

[9] M. H. Shu, C. H. Cheng, and J. R. Chang, "Using intuitionistic fuzzy sets for fault-tree analysis on printed circuit board assembly," Microelectronics Reliability, vol. 46, no. 12, pp. 2139-2148, 2006.

[10] S. Wan and J. Dong, Decision Making Theories and Methods Based on Interval-Valued Intuitionistic Fuzzy Set, Springer, Singapore, 2020.

[11] S. Wan, G. Xu, and J. Dong, "An Atanassov intuitionistic fuzzy programming method for group decision making with interval-valued Atanassov intuitionistic fuzzy preference relations," Applied Soft Computing, vol. 95, Article ID 106556, 2020.

[12] S. Melliani and L. S. Chadli, "Intuitionistic fuzzy differential equation," Notes on Intuitionistic Fuzzy Sets, vol. 6, pp. 37-41, 2000.

[13] B. Ben Amma, S. Melliani, and L. S. Chadli, "The cauchy problem of intuitionistic fuzzy differential equations," Notes on Intuitionistic Fuzzy Sets, vol. 24, no. 1, pp. 37-47, 2018.

[14] S. Melliani, M. Elomari, M. Atraoui, and L. S. Chadli, "Intuitionistic fuzzy differential equation with nonlocal condition," Notes on Intuitionistic Fuzzy Sets, vol. 21, no. 4, pp. 58-68, 2015.

[15] B. Ben Amma, S. Melliani, and L. S. Chadli, Intuitionistic Fuzzy Functional Differential Equations, Fuzzy Logic in Intelligent System Design: Theory and Applications, pp. 335-357, Springer International Publishing, Cham, Switzerland, 2018.

[16] B. Ben Amma, S. Melliani, and L. S. Chadli, "Numerical solution of intuitionistic fuzzy differential equations by Euler and Taylor methods," Notes on Intuitionistic Fuzzy Sets, vol. 22, no. 2, pp. 71-86, 2016.

[17] B. Ben Amma, S. Melliani, and L. S. Chadli, "Numerical solution of intuitionistic fuzzy differential equations by Adams three order predictor-corrector method," Notes on Intuitionistic Fuzzy Sets, vol. 22, no. 3, pp. 47-69, 2016.

[18] M. Mary Jansi Rani and K. Jamshida, "Numerical solution of intuitionistic fuzzy differential equations using Runge-Kutta method," International Journal of Scientific Research and Modern Education, vol. 5, no. 2, pp. 2455-5630, 2020.

[19] S. Melliani, H. Atti, B. Ben Amma, and L. S. Chadli, "Solution of $n$-th order intuitionistic fuzzy differential equation by variational iteration method," Notes on Intuitionistic Fuzzy Sets, vol. 24, pp. 92-105, 2018.

[20] V. Nirmala, V. Parimala, and P. Rajarajeswari, "Multiple numerical solutions for intuitionistic fuzzy differential equations," International Journal of Advanced Research Trends in Engineering and Technology, vol. 5, no. 12, pp. $477-483,2018$.

[21] S. Melliani and L. S. Chadli, "Introduction to intuitionistic fuzzy partial differential equations," Notes on Intuitionistic Fuzzy Sets, vol. 7, pp. 39-42, 2001.

[22] B. Ben Amma, S. Melliani, and L. S. Chadli, "Integral boundary value problem for intuitionistic fuzzy partial hyperbolic differential equations," Nonlinear Analysis and Boundary Value Problems, Springer, vol. 292, Berlin, Germany, 2019. 
[23] B. Ben Amma, S. Melliani, and L. S. Chadli, "The existence and uniqueness of intuitionistic fuzzy solutions for intuitionistic fuzzy partial functional differential equations," International Journal of Differential Equations, vol. 2019, Article ID 9210641, 13 pages, 2019.

[24] S. Bernard, "Moving the boundaries of granulopoiesis modelling," Bulletin of Mathematical Biology, vol. 78, pp. 2358-2363, 2016.

[25] M. Craig, A. R. Humphries, and M. C. Mackey, "A mathematical model of granulopoiesis incorporating the negative feedback dynamics and kinetics of G-CSF/neutrophil binding and internalization," Bulletin of Mathematical Biology, vol. 78, pp. 2304-2357, 2016.

[26] F. A. Davidson and S. A. Gourley, "The effects of temporal delays in a model for a food-limited diffusion population," Journal of Mathematical Analysis and Applications, vol. 261, pp. 633-648, 2001.

[27] F. Hartung, T. Krisztin, H.-O. Walther, and J. Wu, "Chapter 5 functional differential equations with state-dependent delays: theory and applications," Handbook of Differential Equations: Ordinary Differential Equations, Elsevier, vol. 4, pp. 435-545, Amsterdam, Netherlands, 2006.

[28] T. Heil, I. Fischer, W. Elsaer, B. Krauskopf, K. Green, and A. Gavrielides, "Delay dynamics of semiconductor lasers with short external cavities: bifurcation scenarios, and mechanisms," Physical Review E.vol. 67, Article ID 066214, 2003.

[29] J. M. Mahaffy, J. Bélair, and M. C. Mackey, "Hematopoietic model with moving boundary condition and state dependent delay: applications in erythropoiesis," Journal of Theoretical Biology, vol. 190, pp. 135-146, 1998.

[30] A. Otto and G. Radons, "Transformations from variable delays to constant delays with applications in engineering and biology," in Advances in Delays and Dynamics, vol. 7, pp. 169-183, Springer International Publishing, Cham, Switzerland, 2017.

[31] A. V. Rezounenko, "A condition on delay for differential equations with discrete state-dependent delay," Journal of Mathematical Analysis and Applications, vol. 385, pp. 506516, 2012.

[32] J. Sieber and B. Krauskopf, "Complex balancing motions of an inverted pendulum subject to delayed feedback control," Physica D: Nonlinear Phenomena, vol. 197, no. 3, pp. 332-345, 2004.

[33] L. Yongkun, "Periodic solutions for delay Lotka Volterra competition systems," Journal of Mathematical Analysis and Applications, vol. 246, no. 1, pp. 230-244, 2000.

[34] S. Melliani, M. Elomari, L. S. Chadli, and R. Ettoussi, "Intuitionistic fuzzy metric space," Notes on Intuitionistic Fuzzy Sets, vol. 21, no. 1, pp. 43-53, 2015. 